\title{
Nineteen quaternary quadratic forms
}

\author{
by
Ayşe Alaca, Şaban Alaca, Mathieu F. Lemire and Kenneth S. Williams (Ottawa)

1. Introduction. Let $\mathbb{N}, \mathbb{N}_{0}, \mathbb{Z}$ and $\mathbb{C}$ denote the sets of positive integers, nonnegative integers, integers and complex numbers respectively. Throughout this paper $q$ denotes a complex variable such that $|q|<1$. For $a, b, c, d \in \mathbb{N}$ and $n \in \mathbb{N}_{0}$ we define

$$
N(a, b, c, d ; n)=\operatorname{card}\left\{(x, y, z, t) \in \mathbb{Z}^{4} \mid n=a x^{2}+b y^{2}+c z^{2}+d t^{2}\right\} .
$$

Clearly

$$
N(a, b, c, d ; 0)=1 .
$$

In this paper we determine in a uniform manner a formula for $N(a, b, c, d ; n)$, valid for all $n \in \mathbb{N}$, for each of the nineteen quaternary quadratic forms given by

$$
\begin{aligned}
(a, b, c, d)= & (1,1,1,1),(1,1,1,4),(1,1,2,2),(1,1,3,3),(1,1,3,12), \\
& (1,1,4,4),(1,1,6,6),(1,1,12,12),(1,2,2,4),(1,2,3,6), \\
& (1,3,3,4),(1,3,4,12),(1,4,4,4),(1,4,6,6),(1,4,12,12), \\
& (2,2,3,3),(2,2,3,12),(3,3,4,4),(3,4,4,12) .
\end{aligned}
$$

We show that for each of the forms listed in (1.3), formulae for $N(a, b, c, d ; n)$ $(n \in \mathbb{N})$ can be given in terms of the sum of divisors function

$$
\sigma(n)= \begin{cases}\sum_{d \mid n} d, & n \in \mathbb{N}, \\ 0, & n \notin \mathbb{N},\end{cases}
$$

where $d$ runs through the positive integers dividing $n$, and the integers $a(n)$, which are defined for $n \in \mathbb{N}_{0}$ by

2000 Mathematics Subject Classification: 11E20, 11E25.

Key words and phrases: quaternary quadratic forms, theta function.

The fourth author was supported by research grant A-7233 from the Natural Sciences and Engineering Research Council of Canada. 


$$
\sum_{n=0}^{\infty} a(n) q^{n}=q \prod_{n=1}^{\infty}\left(1-q^{2 n}\right)\left(1-q^{4 n}\right)\left(1-q^{6 n}\right)\left(1-q^{12 n}\right),
$$

so that $a(n)=0$ for $n \equiv 0(\bmod 2)$. The formulae for $N(a, b, c, d ; n)$ for the seven quaternary forms given by

$$
\begin{aligned}
(a, b, c, d)= & (1,1,1,1),(1,1,1,4),(1,1,2,2),(1,1,3,3), \\
& (1,1,4,4),(1,2,2,4),(1,4,4,4)
\end{aligned}
$$

depend only on $\sigma(n)$, whereas those for the remaining twelve forms in (1.3) depend upon $\sigma(n)$ and $a(n)$. Numerical evidence suggests that the nineteen forms listed in (1.3) are the only ones satisfying $1 \leq a \leq b \leq c \leq d \leq 12$, $\operatorname{gcd}(a, b, c, d)=1$ and $a, b, c, d \mid 12$ for which $N(a, b, c, d ; n)$ can be expressed in terms of $\sigma(n)$ and $a(n)$ for all $n \in \mathbb{N}$.

We remark that the $q$-series with coefficients $a(n)$ is a cusp form of weight 2 . In terms of Dedekind's eta function $\eta(z)$ we have

$$
\sum_{n=1}^{\infty} a(n) q^{n}=\eta(2 z) \eta(4 z) \eta(6 z) \eta(12 z)
$$

where $q=e^{2 \pi i z}$ and $\operatorname{Re}(z)>0$. It is known that $a(n)$ is a multiplicative function of $n$ [25, p. 4853].

It is also convenient for our purposes to define the integers $b(n)\left(n \in \mathbb{N}_{0}\right)$ by

$$
\sum_{n=0}^{\infty} b(n) q^{n}=\prod_{n=1}^{\infty} \frac{\left(1-q^{2 n}\right)^{10}\left(1-q^{6 n}\right)^{4}}{\left(1-q^{n}\right)^{4}\left(1-q^{4 n}\right)^{4}\left(1-q^{12 n}\right)^{2}},
$$

and the integers $c(n)\left(n \in \mathbb{N}_{0}\right)$ by

$$
\sum_{n=0}^{\infty} c(n) q^{n}=\prod_{n=1}^{\infty} \frac{\left(1-q^{2 n}\right)^{4}\left(1-q^{6 n}\right)^{10}}{\left(1-q^{3 n}\right)^{4}\left(1-q^{4 n}\right)^{2}\left(1-q^{12 n}\right)^{4}} .
$$

Jacobi's theta function $\varphi(q)$ is defined by

$$
\varphi(q)=\sum_{n=-\infty}^{\infty} q^{n^{2}}
$$

Using Jacobi's infinite product expansions

$$
\varphi(q)=\prod_{n=1}^{\infty} \frac{\left(1-q^{2 n}\right)^{5}}{\left(1-q^{n}\right)^{2}\left(1-q^{4 n}\right)^{2}}
$$

and

$$
\varphi(-q)=\prod_{n=1}^{\infty} \frac{\left(1-q^{n}\right)^{2}}{\left(1-q^{2 n}\right)}
$$


we see that

$$
\begin{aligned}
& \prod_{n=1}^{\infty} \frac{\left(1-q^{2 n}\right)^{10}\left(1-q^{6 n}\right)^{4}}{\left(1-q^{n}\right)^{4}\left(1-q^{4 n}\right)^{4}\left(1-q^{12 n}\right)^{2}}=\varphi^{2}(q) \varphi^{2}\left(-q^{6}\right), \\
& \prod_{n=1}^{\infty} \frac{\left(1-q^{2 n}\right)^{4}\left(1-q^{6 n}\right)^{10}}{\left(1-q^{3 n}\right)^{4}\left(1-q^{4 n}\right)^{2}\left(1-q^{12 n}\right)^{4}}=\varphi^{2}\left(-q^{2}\right) \varphi^{2}\left(q^{3}\right),
\end{aligned}
$$

so that for $n \in \mathbb{N}_{0}$,

$$
b(n)=\sum_{\substack{(x, y, z, t) \in \mathbb{Z}^{4} \\ x^{2}+y^{2}+6 z^{2}+6 t^{2}=n}}(-1)^{z+t}, \quad c(n)=\sum_{\substack{(x, y, z, t) \in \mathbb{Z}^{4} \\ 2 x^{2}+2 y^{2}+3 z^{2}+3 t^{2}=n}}(-1)^{x+y} .
$$

Hence $b(n)$ and $c(n)$ can be viewed as "twists" of $N(1,1,6,6 ; n)$ and $N(2,2,3,3 ; n)$ respectively.

The values of $a(n), b(n)$ and $c(n)$ for $n \in\{0,1,2, \ldots, 20\}$ are given in Table 1.

Table 1

\begin{tabular}{rrrr}
\hline$n$ & $a(n)$ & $b(n)$ & $c(n)$ \\
\hline 0 & 0 & 1 & 1 \\
1 & 1 & 4 & 0 \\
2 & 0 & 4 & -4 \\
3 & -1 & 0 & 4 \\
4 & 0 & 4 & 4 \\
5 & -2 & 8 & -16 \\
6 & 0 & -4 & 4 \\
7 & 0 & -16 & 16 \\
8 & 0 & -12 & -12 \\
9 & 1 & 4 & 0 \\
10 & 0 & -8 & 8 \\
11 & 4 & -32 & 16 \\
12 & 0 & 4 & 4 \\
13 & -2 & 24 & -32 \\
14 & 0 & 0 & 0 \\
15 & 2 & -16 & 8 \\
16 & 0 & -12 & -12 \\
17 & 2 & 40 & -32 \\
18 & 0 & 4 & -4 \\
19 & -4 & -32 & 48 \\
20 & 0 & 24 & 24 \\
\hline & & &
\end{tabular}


We now describe how the formulae for $N(a, b, c, d ; n)$ for the nineteen quaternary quadratic forms $a x^{2}+b y^{2}+c z^{2}+d t^{2}$ listed in (1.3) are proved. From (1.8) we see that

$$
\sum_{n=0}^{\infty} N(a, b, c, d ; n) q^{n}=\varphi\left(q^{a}\right) \varphi\left(q^{b}\right) \varphi\left(q^{c}\right) \varphi\left(q^{d}\right) .
$$

Following [4, p. 178] we set

$$
\begin{aligned}
& p=p(q)=\frac{\varphi^{2}(q)-\varphi^{2}\left(q^{3}\right)}{2 \varphi^{2}\left(q^{3}\right)}, \\
& k=k(q)=\frac{\varphi^{3}\left(q^{3}\right)}{\varphi(q)},
\end{aligned}
$$

which, as functions in the variable $z$, are modular forms of weights 0 and 2 . We begin by determining the series expansions of $k^{2}, p k^{2}, p^{2} k^{2}, p^{3} k^{2}$ and $p^{4} k^{2}$ in powers of $q$. We prove the following theorem in Section 2 .

TheOREM 1.1.

$$
\begin{aligned}
k^{2}=1+\sum_{n=1}^{\infty}(-4 \sigma(n)+24 \sigma(n / 2)-4 \sigma(n / 3)-16 \sigma(n / 4) \\
-8 \sigma(n / 6)-16 \sigma(n / 12)) q^{n} .
\end{aligned}
$$

(b) $\quad p k^{2}=\sum_{n=1}^{\infty}(2 \sigma(n)-12 \sigma(n / 2)+6 \sigma(n / 3)+8 \sigma(n / 4)$

$$
+4 \sigma(n / 6)-8 \sigma(n / 12)) q^{n} .
$$

(c) $p^{2} k^{2}=\sum_{n=1}^{\infty}(4 \sigma(n / 2)-8 \sigma(n / 3)+4 \sigma(n / 6)) q^{n}$.

(d) $p^{3} k^{2}=\sum_{n=1}^{\infty}(8 \sigma(n / 3)-8 \sigma(n / 4)-8 \sigma(n / 6)+8 \sigma(n / 12)) q^{n}$.

(e) $\quad p^{4} k^{2}=\sum_{n=1}^{\infty}(16 \sigma(n / 4)-32 \sigma(n / 6)+16 \sigma(n / 12)) q^{n}$.

The following corollary follows immediately from Theorem 1.1.

Corollary 1.1.

(a) $\quad(1+2 p)^{2} k^{2}=1+\sum_{n=1}^{\infty}(4 \sigma(n)-8 \sigma(n / 2)-12 \sigma(n / 3)+16 \sigma(n / 4)$

$$
+24 \sigma(n / 6)-48 \sigma(n / 12)) q^{n} .
$$

(b) $\quad(1+2 p)^{3} k^{2}=1+\sum_{n=1}^{\infty}(8 \sigma(n)-32 \sigma(n / 4)) q^{n}$. 
(c) $\quad(1+2 p)\left(1+p+p^{2}\right) k^{2}$

$$
=1+\sum_{n=1}^{\infty}(2 \sigma(n)+6 \sigma(n / 3)-8 \sigma(n / 4)-24 \sigma(n / 12)) q^{n} .
$$

(d) $\quad(1-p)(1+p)(1+2 p) k^{2}$

$$
=1+\sum_{n=1}^{\infty}(-4 \sigma(n / 2)+16 \sigma(n / 4)+12 \sigma(n / 6)-48 \sigma(n / 12)) q^{n} .
$$

Then in Section 3 we determine the series expansions in powers of $q$ of other functions of $p$ and $k$ for which the coefficients of the powers of $q$ can also be expressed in terms of $\sigma$. Recall that the Legendre-Jacobi-Kronecker symbol for discriminant -4 is defined for $n \in \mathbb{N}$ by

$$
\left(\frac{-4}{n}\right)= \begin{cases}(-1)^{(n-1) / 2} & \text { if } n \text { is odd, } \\ 0 & \text { if } n \text { is even. }\end{cases}
$$

THEOREM 1.2 .

(a)

$$
\begin{aligned}
(1-p)^{3 / 2}(1+p)^{1 / 2}(1+2 p)^{3 / 2} k^{2} & \\
= & 1+\sum_{n=1}^{\infty}(-8 \sigma(n / 2)+48 \sigma(n / 4)-64 \sigma(n / 8)) q^{n} .
\end{aligned}
$$

(b) $\quad(1-p)^{1 / 2}(1+p)^{3 / 2}(1+2 p)^{1 / 2} k^{2}$

$$
=1+\sum_{n=1}^{\infty}(-8 \sigma(n / 6)+48 \sigma(n / 12)-64 \sigma(n / 24)) q^{n} .
$$

(c) $\quad(1-p)^{3 / 4}(1+p)^{1 / 4}(1+2 p)^{9 / 4} k^{2}$

$$
=1+\sum_{n=1}^{\infty}\left(4\left(\frac{-4}{n}\right) \sigma(n)-8 \sigma(n / 4)+48 \sigma(n / 8)-64 \sigma(n / 16)\right) q^{n} .
$$

(d) $\quad(1-p)^{9 / 4}(1+p)^{3 / 4}(1+2 p)^{3 / 4} k^{2}$

$$
=1+\sum_{n=1}^{\infty}\left(-4\left(\frac{-4}{n}\right) \sigma(n)-8 \sigma(n / 4)+48 \sigma(n / 8)-64 \sigma(n / 16)\right) q^{n} .
$$

In Section 4 we determine each of the series $\sum_{n=0}^{\infty} a(n) q^{n}, \sum_{n=0}^{\infty} b(n) q^{n}$ and $\sum_{n=0}^{\infty} c(n) q^{n}$ in terms of $p$ and $k$.

THEOREM 1.3.

$$
\begin{aligned}
\frac{1}{4} p(2+p)(1-p)^{1 / 2}(1+p)^{1 / 2}(1+2 p)^{1 / 2} k^{2} & =\sum_{n=1}^{\infty} a(n) q^{n} . \\
(1-p)^{1 / 4}(1+p)^{3 / 4}(1+2 p)^{7 / 4} k^{2} & =\sum_{n=0}^{\infty} b(n) q^{n} .
\end{aligned}
$$


(c)

$$
\begin{aligned}
& (1-p)^{3 / 4}(1+p)^{1 / 4}(1+2 p)^{5 / 4} k^{2}=\sum_{n=0}^{\infty} c(n) q^{n} . \\
& (1-p)^{7 / 4}(1+p)^{5 / 4}(1+2 p)^{1 / 4} k^{2}=\sum_{n=0}^{\infty} b(n)(-1)^{n} q^{n} . \\
& (1-p)^{5 / 4}(1+p)^{7 / 4}(1+2 p)^{3 / 4} k^{2}=\sum_{n=0}^{\infty} c(n)(-1)^{n} q^{n} .
\end{aligned}
$$

The next theorem shows that each of the quantities

$$
p^{j}(1-p)^{1 / 2}(1+p)^{1 / 2}(1+2 p)^{1 / 2} k^{2}, \quad j \in\{0,1,2\},
$$

has a power series expansion in $q$ with coefficients depending only on $\sigma$ and $a$. This result is proved in Section 5 .

THEOREM 1.4.

(a)

$$
\begin{aligned}
& (1-p)^{1 / 2}(1+p)^{1 / 2}(1+2 p)^{1 / 2} k^{2} \\
& =1+\sum_{n=1}^{\infty}(2 \sigma(n / 2)-12 \sigma(n / 4)-10 \sigma(n / 6)+16 \sigma(n / 8) \\
& \quad+60 \sigma(n / 12)-80 \sigma(n / 24)-2 a(n)) q^{n} .
\end{aligned}
$$

(b) $\quad p(1-p)^{1 / 2}(1+p)^{1 / 2}(1+2 p)^{1 / 2} k^{2}$

$$
\begin{aligned}
=\sum_{n=1}^{\infty}(-2 \sigma(n / 2)+12 \sigma( & n / 4)+2 \sigma(n / 6)-16 \sigma(n / 8) \\
& -12 \sigma(n / 12)+16 \sigma(n / 24)+2 a(n)) q^{n} .
\end{aligned}
$$

(c) $\quad p^{2}(1-p)^{1 / 2}(1+p)^{1 / 2}(1+2 p)^{1 / 2} k^{2}$

$$
\begin{aligned}
=\sum_{n=1}^{\infty}(4 \sigma(n / 2)-24 \sigma(n / 4)-4 \sigma & (n / 6)+32 \sigma(n / 8) \\
& +24 \sigma(n / 12)-32 \sigma(n / 24)) q^{n} .
\end{aligned}
$$

As

$$
\begin{aligned}
(1-p)^{1 / 2}(1+p)^{1 / 2}(1+2 p)^{3 / 2}= & (1-p)^{1 / 2}(1+p)^{1 / 2}(1+2 p)^{1 / 2} \\
& +2 p(1-p)^{1 / 2}(1+p)^{1 / 2}(1+2 p)^{1 / 2}, \\
(1-p)^{1 / 2}(1+p)^{3 / 2}(1+2 p)^{3 / 2}= & (1-p)^{1 / 2}(1+p)^{1 / 2}(1+2 p)^{1 / 2} \\
& +3 p(1-p)^{1 / 2}(1+p)^{1 / 2}(1+2 p)^{1 / 2} \\
& +2 p^{2}(1-p)^{1 / 2}(1+p)^{1 / 2}(1+2 p)^{1 / 2}, \\
(1-p)^{3 / 2}(1+p)^{1 / 2}(1+2 p)^{1 / 2}= & (1-p)^{1 / 2}(1+p)^{1 / 2}(1+2 p)^{1 / 2} \\
& -p(1-p)^{1 / 2}(1+p)^{1 / 2}(1+2 p)^{1 / 2},
\end{aligned}
$$

we obtain the following result from Theorem 1.4. 
COROllary 1.2 .

(a) $\quad(1-p)^{1 / 2}(1+p)^{3 / 2}(1+2 p)^{3 / 2} k^{2}$

$$
\begin{aligned}
=1+\sum_{n=1}^{\infty}(4 \sigma(n / 2)-34 \sigma( & n / 4)-12 \sigma(n / 6)+32 \sigma(n / 8) \\
+ & 72 \sigma(n / 12)-96 \sigma(n / 24)+4 a(n)) q^{n} .
\end{aligned}
$$

(b) $\quad(1-p)^{1 / 2}(1+p)^{1 / 2}(1+2 p)^{3 / 2} k^{2}$

$$
\begin{aligned}
=1+\sum_{n=1}^{\infty}(-2 \sigma(n / 2)+12 \sigma & (n / 4)-6 \sigma(n / 6)-16 \sigma(n / 8) \\
& +36 \sigma(n / 12)-48 \sigma(n / 24)+2 a(n)) q^{n} .
\end{aligned}
$$

(c) $\quad(1-p)^{3 / 2}(1+p)^{1 / 2}(1+2 p)^{1 / 2} k^{2}$

$$
\begin{aligned}
=1+\sum_{n=1}^{\infty}(4 \sigma(n / 2)-24 \sigma( & n / 4)-12 \sigma(n / 6)+32 \sigma(n / 8) \\
+ & 72 \sigma(n / 12)-96 \sigma(n / 24)-4 a(n)) q^{n} .
\end{aligned}
$$

Our next theorem, which is proved in [2, Theorem 2.4], expresses each of $\varphi\left(q^{k}\right)(k \in\{1,2,3,4,6,12\})$ in terms of $p$ and $k$.

THEOREM 1.5 .

$$
\begin{aligned}
\varphi(q) & =(1+2 p)^{3 / 4} k^{1 / 2}, \\
\varphi\left(q^{2}\right) & =\frac{1}{\sqrt{2}}\left((1+2 p)^{3 / 2}+(1-p)^{3 / 2}(1+p)^{1 / 2}\right)^{1 / 2} k^{1 / 2}, \\
\varphi\left(q^{3}\right) & =(1+2 p)^{1 / 4} k^{1 / 2}, \\
\varphi\left(q^{4}\right) & =\frac{1}{2}\left((1+2 p)^{3 / 4}+(1-p)^{3 / 4}(1+p)^{1 / 4}\right) k^{1 / 2}, \\
\varphi\left(q^{6}\right) & =\frac{1}{\sqrt{2}}\left((1+2 p)^{1 / 2}+(1-p)^{1 / 2}(1+p)^{3 / 2}\right)^{1 / 2} k^{1 / 2} \\
\varphi\left(q^{12}\right) & =\frac{1}{2}\left((1+2 p)^{1 / 4}+(1-p)^{1 / 4}(1+p)^{3 / 4}\right) k^{1 / 2} .
\end{aligned}
$$

From Theorem 1.5(b)(e) we deduce

$$
\varphi\left(q^{2}\right) \varphi\left(q^{6}\right)=\frac{1}{2}\left(1+p+p^{2}+(1-p)^{1 / 2}(1+p)^{1 / 2}(1+2 p)^{1 / 2}\right) k .
$$

We are now in a position to determine a formula for $N(a, b, c, d ; n)$ $(n \in \mathbb{N})$ for each of the nineteen forms given in (1.3). For each $(a, b, c, d)$ in (1.3) we use (1.9) and Theorem 1.5 to express $\sum_{n=0}^{\infty} N(a, b, c, d ; n) q^{n}$ as a function of $p$ and $k$. Then, using Theorems 1.1-1.4 and Corollaries 1.1 and 1.2, we express this function in terms of $\sum_{n=0}^{\infty} \sigma(n) q^{k n}(k \in\{1,2,3$, $4,6,12\}), \sum_{n=0}^{\infty} a(n) q^{n}, \sum_{n=0}^{\infty} b(n) q^{n}$ and $\sum_{n=0}^{\infty} c(n) q^{n}$. The formula for $N(a, b, c, d ; n)$ is then found by equating coefficients of $q^{n}(n \in \mathbb{N})$. Each such formula depends at most on $\sigma, a, b$ and $c$. The details are carried out 
in Section 6. The formulae are given in Theorems 1.6-1.24. In the course of the proofs of these theorems, we obtain some relationships between $a(n)$, $b(n)$ and $c(n)$. These are stated in Theorem 1.25. Finally, we use Theorem 1.25 to give all the formulae of Theorems 1.6-1.24 in terms only of $\sigma$ and $a$. The reader is advised that the final form of those theorems marked with an asterisk is given after Theorem 1.25.

Theorem 1.6. Let $n \in \mathbb{N}$. Set $n=2^{\alpha} N$, where $\alpha \in \mathbb{N}_{0}, N \in \mathbb{N}$ and $\operatorname{gcd}(N, 2)=1$. Then

$$
N(1,1,1,1 ; n)= \begin{cases}8 \sigma(N) & \text { if } n \equiv 1(\bmod 2), \\ 24 \sigma(N) & \text { if } n \equiv 0(\bmod 2) .\end{cases}
$$

This is Jacobi's famous formula for the number of representations of a positive integer as the sum of four squares.

Theorem 1.7. Let $n \in \mathbb{N}$. Set $n=2^{\alpha} N$, where $\alpha \in \mathbb{N}_{0}, N \in \mathbb{N}$ and $\operatorname{gcd}(N, 2)=1$. Then

$$
N(1,1,1,4 ; n)= \begin{cases}\left(4+2(-1)^{(N-1) / 2}\right) \sigma(N) & \text { if } n \equiv 1(\bmod 2), \\ 12 \sigma(N) & \text { if } n \equiv 2(\bmod 4), \\ 8 \sigma(N) & \text { if } n \equiv 4(\bmod 8), \\ 24 \sigma(N) & \text { if } n \equiv 0(\bmod 8) .\end{cases}
$$

This formula was stated without proof by Liouville [20] and proved by Pepin [27].

Theorem 1.8. Let $n \in \mathbb{N}$. Set $n=2^{\alpha} N$, where $\alpha \in \mathbb{N}_{0}, N \in \mathbb{N}$ and $\operatorname{gcd}(N, 2)=1$. Then

$$
N(1,1,2,2 ; n)= \begin{cases}4 \sigma(N) & \text { if } n \equiv 1(\bmod 2), \\ 8 \sigma(N) & \text { if } n \equiv 2(\bmod 4), \\ 24 \sigma(N) & \text { if } n \equiv 0(\bmod 4) .\end{cases}
$$

This formula was stated without proof by Liouville [17] and proved by Pepin [26], [27], Bachmann [8], and Deutsch [10].

Theorem 1.9. Let $n \in \mathbb{N}$. Set $n=2^{\alpha} 3^{\beta} N$, where $\alpha, \beta \in \mathbb{N}_{0}, N \in \mathbb{N}$ and $\operatorname{gcd}(N, 6)=1$. Then

$$
N(1,1,3,3 ; n)= \begin{cases}4 \sigma(N) & \text { if } n \equiv 1(\bmod 2), \\ 4\left(2^{\alpha+1}-3\right) \sigma(N) & \text { if } n \equiv 0(\bmod 2) .\end{cases}
$$

This formula was stated without proof by Liouville [16] (see also [22]) and proved by Pepin [27], Bachmann [8] and Kloosterman [14].

Theorem 1.10* Let $n \in \mathbb{N}$. Set $n=2^{\alpha} 3^{\beta} N$, where $\alpha, \beta \in \mathbb{N}_{0}, N \in \mathbb{N}$ and $\operatorname{gcd}(N, 6)=1$. Then 


$$
N(1,1,3,12 ; n)= \begin{cases}2 \sigma(N)+\frac{1}{2} b(n) & \text { if } n \equiv 1(\bmod 2), \\ 2\left(2^{\alpha+1}-3\right) \sigma(N)+\frac{1}{2} b(n) & \text { if } n \equiv 0(\bmod 2) .\end{cases}
$$

Liouville did not consider this form.

Theorem 1.11. Let $n \in \mathbb{N}$. Set $n=2^{\alpha} N$, where $\alpha \in \mathbb{N}_{0}, N \in \mathbb{N}$ and $\operatorname{gcd}(N, 2)=1$. Then

$$
N(1,1,4,4 ; n)= \begin{cases}4 \sigma(N) & \text { if } n \equiv 1(\bmod 4), \\ 0 & \text { if } n \equiv 3(\bmod 4), \\ 4 \sigma(N) & \text { if } n \equiv 2(\bmod 4), \\ 8 \sigma(N) & \text { if } n \equiv 4(\bmod 8), \\ 24 \sigma(N) & \text { if } n \equiv 0(\bmod 8) .\end{cases}
$$

This formula was stated without proof by Liouville [18] and proved by Pepin [26], [27] and Bachmann [8, p. 417].

Theorem 1.12. Let $n \in \mathbb{N}$. Set $n=2^{\alpha} 3^{\beta} N$, where $\alpha, \beta \in \mathbb{N}_{0}, N \in \mathbb{N}$ and $\operatorname{gcd}(N, 6)=1$. Then

$$
N(1,1,6,6 ; n)= \begin{cases}2 \sigma(N)+2 a(n) & \text { if } n \equiv 1(\bmod 2), \\ 4 \sigma(N) & \text { if } n \equiv 2(\bmod 4), \\ 4\left(2^{\alpha}-3\right) \sigma(N) & \text { if } n \equiv 0(\bmod 4) .\end{cases}
$$

This form has been considered by Liouville [24], Kloosterman [14], Gongadze [11] and Köhler [15].

Theorem 1.13* Let $n \in \mathbb{N}$. Set $n=2^{\alpha} 3^{\beta} N$, where $\alpha, \beta \in \mathbb{N}_{0}, N \in \mathbb{N}$ and $\operatorname{gcd}(N, 6)=1$. Then

$$
N(1,1,12,12 ; n)= \begin{cases}\sigma(N)+a(n)+\frac{1}{2} b(n) & \text { if } n \equiv 1(\bmod 4), \\ 0 & \text { if } n \equiv 3(\bmod 4), \\ 2 \sigma(N)+\frac{1}{2} b(n) & \text { if } n \equiv 2(\bmod 4), \\ 2\left(2^{\alpha}-3\right) \sigma(N)+\frac{1}{2} b(n) & \text { if } n \equiv 0(\bmod 4) .\end{cases}
$$

This form was not considered by Liouville.

Theorem 1.14. Let $n \in \mathbb{N}$. Set $n=2^{\alpha} N$, where $\alpha \in \mathbb{N}_{0}, N \in \mathbb{N}$ and $\operatorname{gcd}(N, 2)=1$. Then

$$
N(1,2,2,4 ; n)= \begin{cases}2 \sigma(N) & \text { if } n \equiv 1(\bmod 2) \\ 4 \sigma(N) & \text { if } n \equiv 2(\bmod 4) \\ 8 \sigma(N) & \text { if } n \equiv 4(\bmod 8) \\ 24 \sigma(N) & \text { if } n \equiv 0(\bmod 8)\end{cases}
$$

This form has been treated by Liouville [21], Pepin [26, p. 177], [27, pp. 31, 42] and Bachmann [8, p. 418]. 
Theorem 1.15. Let $n \in \mathbb{N}$. Set $n=2^{\alpha} 3^{\beta} N$, where $\alpha, \beta \in \mathbb{N}_{0}, N \in \mathbb{N}$ and $\operatorname{gcd}(N, 6)=1$. Then

$$
N(1,2,3,6 ; n)= \begin{cases}\left(3^{\beta+1}-2\right) \sigma(N)+a(n) & \text { if } n \equiv 1(\bmod 2), \\ 2\left(3^{\beta+1}-2\right) \sigma(N) & \text { if } n \equiv 2(\bmod 4), \\ 6\left(3^{\beta+1}-2\right) \sigma(N) & \text { if } n \equiv 0(\bmod 4) .\end{cases}
$$

This form has been considered by Liouville [23], Bachmann [8, p. 423], Griffiths [12], [13], Gongadze [11] and Köhler [15].

Theorem 1.16* Let $n \in \mathbb{N}$. Set $n=2^{\alpha} 3^{\beta} N$, where $\alpha, \beta \in \mathbb{N}_{0}, N \in \mathbb{N}$ and $\operatorname{gcd}(N, 6)=1$. Then

$$
N(1,3,3,4 ; n)= \begin{cases}2 \sigma(N)+\frac{1}{2} c(n) & \text { if } n \equiv 1(\bmod 2), \\ 2\left(2^{\alpha+1}-3\right) \sigma(N)+\frac{1}{2} c(n) & \text { if } n \equiv 0(\bmod 2) .\end{cases}
$$

This form was not treated by Liouville.

Theorem 1.17. Let $n \in \mathbb{N}$. Set $n=2^{\alpha} 3^{\beta} N$, where $\alpha, \beta \in \mathbb{N}_{0}, N \in \mathbb{N}$ and $\operatorname{gcd}(N, 6)=1$. Then

$$
N(1,3,4,12 ; n)= \begin{cases}\sigma(N)+\frac{1}{4} b(n)+\frac{1}{4} c(n) & \text { if } n \equiv 1(\bmod 2), \\ 0 & \text { if } n \equiv 2(\bmod 4), \\ 6\left(2^{\alpha-1}-1\right) \sigma(N)+\frac{1}{4} b(n)+\frac{1}{4} c(n) & \text { if } n \equiv 0(\bmod 4) .\end{cases}
$$

Elementary remarks on this form were given by Liouville [19] and Pepin [27, p. 24].

Theorem 1.18. Let $n \in \mathbb{N}$. Set $n=2^{\alpha} N$, where $\alpha \in \mathbb{N}_{0}, N \in \mathbb{N}$ and $\operatorname{gcd}(N, 2)=1$. Then

$$
N(1,4,4,4 ; n)= \begin{cases}2 \sigma(N) & \text { if } n \equiv 1(\bmod 4), \\ 0 & \text { if } n \equiv 2,3(\bmod 4) \\ 8 \sigma(N) & \text { if } n \equiv 4(\bmod 8) \\ 24 \sigma(N) & \text { if } n \equiv 0(\bmod 8) .\end{cases}
$$

This result was stated by Liouville without proof in [20] and proved by Pepin [27, p. 43].

Theorem 1.19* Let $n \in \mathbb{N}$. Set $n=2^{\alpha} 3^{\beta} N$, where $\alpha, \beta \in \mathbb{N}_{0}, N \in \mathbb{N}$ and $\operatorname{gcd}(N, 6)=1$. Then

$$
N(1,4,6,6 ; n)= \begin{cases}\sigma(N)+a(n) & \text { if } n \equiv 1(\bmod 2), \\ 2 \sigma(N)+\frac{1}{2} c(n) & \text { if } n \equiv 2(\bmod 4), \\ 2\left(2^{\alpha}-3\right) \sigma(N)+\frac{1}{2} c(n) & \text { if } n \equiv 0(\bmod 4) .\end{cases}
$$

Liouville did not consider this form. 
Theorem 1.20* Let $n \in \mathbb{N}$. Set $n=2^{\alpha} 3^{\beta} N$, where $\alpha, \beta \in \mathbb{N}_{0}, N \in \mathbb{N}$ and $\operatorname{gcd}(N, 6)=1$. Then

$$
N(1,4,12,12 ; n)= \begin{cases}\frac{1}{2} \sigma(N)+\frac{1}{2} a(n)+\frac{1}{4} b(n) & \text { if } n \equiv 1(\bmod 4), \\ 0 & \text { if } n \equiv 2,3(\bmod 4), \\ 2\left(2^{\alpha}-3\right) \sigma(N)+\frac{1}{4} b(n)+\frac{1}{4} c(n) & \text { if } n \equiv 0(\bmod 4) .\end{cases}
$$

Liouville did not consider this form.

Theorem 1.21. Let $n \in \mathbb{N}$. Set $n=2^{\alpha} 3^{\beta} N$, where $\alpha, \beta \in \mathbb{N}_{0}, N \in \mathbb{N}$ and $\operatorname{gcd}(N, 6)=1$. Then

$$
N(2,2,3,3 ; n)= \begin{cases}2 \sigma(N)-2 a(n) & \text { if } n \equiv 1(\bmod 2), \\ 4 \sigma(N) & \text { if } n \equiv 2(\bmod 4), \\ 4\left(2^{\alpha}-3\right) \sigma(N) & \text { if } n \equiv 0(\bmod 4) .\end{cases}
$$

Partial results were stated by Liouville [24] without proof.

Theorem 1.22* Let $n \in \mathbb{N}$. Set $n=2^{\alpha} 3^{\beta} N$, where $\alpha, \beta \in \mathbb{N}_{0}, N \in \mathbb{N}$ and $\operatorname{gcd}(N, 6)=1$. Then

$$
N(2,2,3,12 ; n)= \begin{cases}\sigma(N)-a(n) & \text { if } n \equiv 1(\bmod 2), \\ 2 \sigma(N)+\frac{1}{2} b(n) & \text { if } n \equiv 2(\bmod 4), \\ 2\left(2^{\alpha}-3\right) \sigma(N)+\frac{1}{2} b(n) & \text { if } n \equiv 0(\bmod 4) .\end{cases}
$$

Liouville did not consider this form.

Theorem 1.23. Let $n \in \mathbb{N}$. Set $n=2^{\alpha} 3^{\beta} N$, where $\alpha, \beta \in \mathbb{N}_{0}, N \in \mathbb{N}$ and $\operatorname{gcd}(N, 6)=1$. Then

$$
N(3,3,4,4 ; n)= \begin{cases}\sigma(N)-a(n)+\frac{1}{2} c(n) & \text { if } n \equiv 3(\bmod 4), \\ 0 & \text { if } n \equiv 1(\bmod 4), \\ 2 \sigma(N)+\frac{1}{2} c(n) & \text { if } n \equiv 2(\bmod 4), \\ 2\left(2^{\alpha}-3\right) \sigma(N)+\frac{1}{2} c(n) & \text { if } n \equiv 0(\bmod 4) .\end{cases}
$$

Liouville did not treat this form.

Theorem 1.24*. Let $n \in \mathbb{N}$. Set $n=2^{\alpha} 3^{\beta} N$, where $\alpha, \beta \in \mathbb{N}_{0}, N \in \mathbb{N}$ and $\operatorname{gcd}(N, 6)=1$. Then

$$
N(3,4,4,12 ; n)= \begin{cases}\frac{1}{2} \sigma(N)-\frac{1}{2} a(n)+\frac{1}{4} c(n) & \text { if } n \equiv 3(\bmod 4), \\ 0 & \text { if } n \equiv 1,2(\bmod 4), \\ 2\left(2^{\alpha}-3\right) \sigma(N)+\frac{1}{4} b(n)+\frac{1}{4} c(n) & \text { if } n \equiv 0(\bmod 4) .\end{cases}
$$

This form was not considered by Liouville.

In the course of proving Theorems 1.6-1.24 we establish the following result.

Theorem 1.25. Let $n \in \mathbb{N}$. Set $n=2^{\alpha} 3^{\beta} N$, where $\alpha, \beta \in \mathbb{N}_{0}, N \in \mathbb{N}$ and $\operatorname{gcd}(N, 6)=1$. Then 
(a)

$$
\begin{array}{ll}
b(n)=2 \sigma(N)+2 a(n) & \text { if } n \equiv 1(\bmod 4), \\
b(n)=-2 \sigma(N)-2 a(n) & \text { if } n \equiv 3(\bmod 4), \\
c(n)=-2 \sigma(N)+2 a(n) & \text { if } n \equiv 1(\bmod 4), \\
c(n)=2 \sigma(N)-2 a(n), & \text { if } n \equiv 3(\bmod 4), \\
b(n)=-c(n)=4 a(n / 2) & \text { if } n \equiv 2(\bmod 4), \\
b(n)=c(n)=4 \sigma(N) & \text { if } n \equiv 4(\bmod 8), \\
b(n)=c(n)=-12 \sigma(N) & \text { if } n \equiv 0(\bmod 8) .
\end{array}
$$

Applying Theorem 1.25 to Theorems $1.10^{*}, 1.13^{*}, 1.16^{*}, 1.17^{*}, 1.19^{*}$, $1.20^{*}, 1.22^{*}, 1.23^{*}$ and $1.24^{*}$, we obtain formulae which do not depend upon $b$ and $c$.

Theorem 1.10. Let $n \in \mathbb{N}$. Set $n=2^{\alpha} 3^{\beta} N$, where $\alpha, \beta \in \mathbb{N}_{0}, N \in \mathbb{N}$ and $\operatorname{gcd}(N, 6)=1$. Then

$$
N(1,1,3,12 ; n)= \begin{cases}3 \sigma(N)+a(n) & \text { if } n \equiv 1(\bmod 4), \\ \sigma(N)-a(n) & \text { if } n \equiv 3(\bmod 4), \\ 2 \sigma(N)+2 a(n / 2) & \text { if } n \equiv 2(\bmod 4), \\ 12 \sigma(N) & \text { if } n \equiv 4(\bmod 8), \\ 4\left(2^{\alpha}-3\right) \sigma(N) & \text { if } n \equiv 0(\bmod 8) .\end{cases}
$$

Theorem 1.13. Let $n \in \mathbb{N}$. Set $n=2^{\alpha} 3^{\beta} N$, where $\alpha, \beta \in \mathbb{N}_{0}, N \in \mathbb{N}$ and $\operatorname{gcd}(N, 6)=1$. Then

$$
N(1,1,12,12 ; n)= \begin{cases}2 \sigma(N)+2 a(n) & \text { if } n \equiv 1(\bmod 4), \\ 0 & \text { if } n \equiv 3(\bmod 4), \\ 2 \sigma(N)+2 a(n / 2) & \text { if } n \equiv 2(\bmod 4), \\ 4 \sigma(N) & \text { if } n \equiv 4(\bmod 8), \\ 4\left(2^{\alpha-1}-3\right) \sigma(N) & \text { if } n \equiv 0(\bmod 8) .\end{cases}
$$

Theorem 1.16. Let $n \in \mathbb{N}$. Set $n=2^{\alpha} 3^{\beta} N$, where $\alpha, \beta \in \mathbb{N}_{0}, N \in \mathbb{N}$ and $\operatorname{gcd}(N, 6)=1$. Then

$$
N(1,3,3,4 ; n)= \begin{cases}\sigma(N)+a(n) & \text { if } n \equiv 1(\bmod 4), \\ 3 \sigma(N)-a(n) & \text { if } n \equiv 3(\bmod 4), \\ 2 \sigma(N)-2 a(n / 2) & \text { if } n \equiv 2(\bmod 4), \\ 12 \sigma(N) & \text { if } n \equiv 4(\bmod 8), \\ 4\left(2^{\alpha}-3\right) \sigma(N) & \text { if } n \equiv 0(\bmod 8) .\end{cases}
$$

Theorem 1.17. Let $n \in \mathbb{N}$. Set $n=2^{\alpha} 3^{\beta} N$, where $\alpha, \beta \in \mathbb{N}_{0}, N \in \mathbb{N}$ and $\operatorname{gcd}(N, 6)=1$. Then 


$$
N(1,3,4,12 ; n)= \begin{cases}\sigma(N)+a(n) & \text { if } n \equiv 1(\bmod 4), \\ \sigma(N)-a(n) & \text { if } n \equiv 3(\bmod 4), \\ 0 & \text { if } n \equiv 2(\bmod 4), \\ 8 \sigma(N) & \text { if } n \equiv 4(\bmod 8), \\ 12\left(2^{\alpha-2}-1\right) \sigma(N) & \text { if } n \equiv 0(\bmod 8) .\end{cases}
$$

Theorem 1.19. Let $n \in \mathbb{N}$. Set $n=2^{\alpha} 3^{\beta} N$, where $\alpha, \beta \in \mathbb{N}_{0}, N \in \mathbb{N}$ and $\operatorname{gcd}(N, 6)=1$. Then

$$
N(1,4,6,6 ; n)= \begin{cases}\sigma(N)+a(n) & \text { if } n \equiv 1(\bmod 2), \\ 2 \sigma(N)-2 a(n / 2) & \text { if } n \equiv 2(\bmod 4), \\ 4 \sigma(N) & \text { if } n \equiv 4(\bmod 8), \\ 4\left(2^{\alpha-1}-3\right) \sigma(N) & \text { if } n \equiv 0(\bmod 8) .\end{cases}
$$

Theorem 1.20. Let $n \in \mathbb{N}$. Set $n=2^{\alpha} 3^{\beta} N$, where $\alpha, \beta \in \mathbb{N}_{0}, N \in \mathbb{N}$ and $\operatorname{gcd}(N, 6)=1$. Then

$$
N(1,4,12,12 ; n)= \begin{cases}\sigma(N)+a(n) & \text { if } n \equiv 1(\bmod 4), \\ 0 & \text { if } n \equiv 2,3(\bmod 4), \\ 4 \sigma(N) & \text { if } n \equiv 4(\bmod 8), \\ 4\left(2^{\alpha-1}-3\right) \sigma(N) & \text { if } n \equiv 0(\bmod 8) .\end{cases}
$$

TheOREM 1.22. Let $n \in \mathbb{N}$. Set $n=2^{\alpha} 3^{\beta} N$, where $\alpha, \beta \in \mathbb{N}_{0}, N \in \mathbb{N}$ and $\operatorname{gcd}(N, 6)=1$. Then

$$
N(2,2,3,12 ; n)= \begin{cases}\sigma(N)-a(n) & \text { if } n \equiv 1(\bmod 2), \\ 2 \sigma(N)+2 a(n / 2) & \text { if } n \equiv 2(\bmod 4), \\ 4 \sigma(N) & \text { if } n \equiv 4(\bmod 8), \\ 4\left(2^{\alpha-1}-3\right) \sigma(N) & \text { if } n \equiv 0(\bmod 8) .\end{cases}
$$

TheOREM 1.23. Let $n \in \mathbb{N}$. Set $n=2^{\alpha} 3^{\beta} N$, where $\alpha, \beta \in \mathbb{N}_{0}, N \in \mathbb{N}$ and $\operatorname{gcd}(N, 6)=1$. Then

$$
N(3,3,4,4 ; n)= \begin{cases}0 & \text { if } n \equiv 1(\bmod 4), \\ 2 \sigma(N)-2 a(n) & \text { if } n \equiv 3(\bmod 4), \\ 2 \sigma(N)-2 a(n / 2) & \text { if } n \equiv 2(\bmod 4), \\ 4 \sigma(N) & \text { if } n \equiv 4(\bmod 8), \\ 4\left(2^{\alpha-1}-3\right) \sigma(N) & \text { if } n \equiv 0(\bmod 8) .\end{cases}
$$

TheOREM 1.24. Let $n \in \mathbb{N}$. Set $n=2^{\alpha} 3^{\beta} N$, where $\alpha, \beta \in \mathbb{N}_{0}, N \in \mathbb{N}$ and $\operatorname{gcd}(N, 6)=1$. Then 


$$
N(3,4,4,12 ; n)= \begin{cases}0 & \text { if } n \equiv 1,2(\bmod 4), \\ \sigma(N)-a(n) & \text { if } n \equiv 3(\bmod 4), \\ 4 \sigma(N) & \text { if } n \equiv 4(\bmod 8), \\ 4\left(2^{\alpha-1}-3\right) \sigma(N) & \text { if } n \equiv 0(\bmod 8) .\end{cases}
$$

The authors have treated other quaternary quadratic forms in [1]-[4], [6] and [28]. These forms require functions other than $\sigma(n)$ and $a(n)$ for their evaluation.

2. Proof of Theorem 1.1. We begin by recalling the duplication, triplication and change of sign principles for $p$ and $k$, which were proved in $[4$, Theorems 9, 10, 11].

Theorem 2.1 (Duplication principle).

$$
\begin{aligned}
& p\left(q^{2}\right)=\frac{1+p-p^{2}-((1-p)(1+p)(1+2 p))^{1 / 2}}{p^{2}}, \\
& k\left(q^{2}\right)=\frac{\left(1+p-p^{2}+((1-p)(1+p)(1+2 p))^{1 / 2}\right) k}{2} .
\end{aligned}
$$

Theorem 2.2 (Triplication principle).

$$
\begin{aligned}
p\left(q^{3}\right)= & 3^{-1}\left(\left(-4-3 p+6 p^{2}+4 p^{3}\right)\right. \\
& +2^{2 / 3}\left(1-2 p-2 p^{2}\right)((1-p)(1+2 p)(2+p))^{1 / 3} \\
& \left.+2^{1 / 3}(1+2 p)((1-p)(1+2 p)(2+p))^{2 / 3}\right), \\
k\left(q^{3}\right)= & 3^{-2}\left(3+2^{2 / 3}(1+2 p)((1-p)(1+2 p)(2+p))^{1 / 3}\right. \\
& \left.+2^{4 / 3}((1-p)(1+2 p)(2+p))^{2 / 3}\right) k .
\end{aligned}
$$

Theorem 2.3 (Change of sign principle).

$$
p(-q)=\frac{-p}{1+p}, \quad k(-q)=(1+p)^{2} k .
$$

The Eisenstein series $L(q)$ is defined by

$$
L(q)=1-24 \sum_{n=1}^{\infty} \sigma(n) q^{n} .
$$

We define

$$
\begin{aligned}
& A(q):=2 L\left(q^{2}\right)-L(q)=1+24 \sum_{n=1}^{\infty}(\sigma(n)-2 \sigma(n / 2)) q^{n}, \\
& B(q):=3 L\left(q^{3}\right)-L(q)=2+24 \sum_{n=1}^{\infty}(\sigma(n)-3 \sigma(n / 3)) q^{n} .
\end{aligned}
$$

Proof of Theorem 1.1. It was shown in [7, eq. (3.84)] that

$$
A(q)=\left(1+14 p+24 p^{2}+14 p^{3}+p^{4}\right) k^{2} .
$$


Applying the duplication principle (Theorem 2.1) to (2.4), we obtain

$$
A\left(q^{2}\right)=\left(1+2 p+6 p^{2}+5 p^{3}-\frac{1}{2} p^{4}\right) k^{2} .
$$

From [7, eq. (3.87)] we have

$$
B(q)=\left(2+16 p+36 p^{2}+16 p^{3}+2 p^{4}\right) k^{2} .
$$

Applying the duplication principle (Theorem 1.1) twice to (2.6), we deduce

$$
\begin{aligned}
& B\left(q^{2}\right)=\left(2+4 p+6 p^{2}+4 p^{3}+2 p^{4}\right) k^{2}, \\
& B\left(q^{4}\right)=\left(2+4 p-2 p^{3}+\frac{1}{2} p^{4}\right) k^{2} .
\end{aligned}
$$

From (2.4)-(2.8) we obtain

$$
\begin{aligned}
k^{2} & =-\frac{2}{9} A(q)+\frac{4}{9} A\left(q^{2}\right)+\frac{1}{18} B(q)+\frac{1}{9} B\left(q^{2}\right)+\frac{2}{9} B\left(q^{4}\right), \\
p k^{2} & =\frac{1}{6} A(q)-\frac{1}{9} A\left(q^{2}\right)-\frac{1}{12} B(q)-\frac{1}{18} B\left(q^{2}\right)+\frac{1}{9} B\left(q^{4}\right), \\
p^{2} k^{2} & =-\frac{1}{9} A(q)+\frac{1}{9} B(q)-\frac{1}{18} B\left(q^{2}\right), \\
p^{3} k^{2} & =\frac{1}{9} A(q)+\frac{1}{9} A\left(q^{2}\right)-\frac{1}{9} B(q)+\frac{1}{9} B\left(q^{2}\right)-\frac{1}{9} B\left(q^{4}\right), \\
p^{4} k^{2} & =-\frac{4}{9} A\left(q^{2}\right)+\frac{4}{9} B\left(q^{2}\right)-\frac{2}{9} B\left(q^{4}\right) .
\end{aligned}
$$

Appealing to (2.2) and (2.3) we obtain the assertions of Theorem 1.1.

3. Proof of Theorem 1.2. Applying the duplication principle to (2.5) we have

$$
\begin{aligned}
A\left(q^{4}\right)= & \left(\frac{1}{4}+\frac{1}{2} p+\frac{3}{2} p^{2}+\frac{5}{4} p^{3}-\frac{1}{8} p^{4}\right) k^{2} \\
& +\frac{3}{4}(1-p)^{3 / 2}(1+p)^{1 / 2}(1+2 p)^{3 / 2} k^{2} .
\end{aligned}
$$

From (3.1), (2.5) and (2.2), we obtain

$$
\begin{aligned}
(1-p)^{3 / 2}(1+p)^{1 / 2} & (1+2 p)^{3 / 2} k^{2} \\
& =\frac{4}{3} A\left(q^{4}\right)-\frac{1}{3} A\left(q^{2}\right)=\frac{8}{3} L\left(q^{8}\right)-2 L\left(q^{4}\right)+\frac{1}{3} L\left(q^{2}\right) \\
& =1+\sum_{n=1}^{\infty}(-8 \sigma(n / 2)+48 \sigma(n / 4)-64 \sigma(n / 8)) q^{n},
\end{aligned}
$$

which is part (a). 
Next, applying the duplication principle to (3.1), we obtain

$$
\begin{aligned}
A\left(q^{8}\right)= & \left(\frac{1}{16}+\frac{1}{8} p+\frac{3}{8} p^{2}+\frac{5}{16} p^{3}-\frac{1}{32} p^{4}\right) k^{2} \\
& +\frac{3}{16}(1-p)^{3 / 2}(1+p)^{1 / 2}(1+2 p)^{3 / 2} k^{2} \\
& +\frac{3}{8}(1-p)^{3 / 4}(1+p)^{1 / 4}(1+2 p)^{9 / 4} k^{2} \\
& +\frac{3}{8}(1-p)^{9 / 4}(1+p)^{3 / 4}(1+2 p)^{3 / 4} k^{2}
\end{aligned}
$$

and to (2.8) we obtain

$$
\begin{aligned}
B\left(q^{8}\right)= & \left(\frac{5}{4}+\frac{5}{2} p-\frac{3}{4} p^{2}-2 p^{3}+\frac{1}{8} p^{4}\right) k^{2} \\
& -\frac{3}{8}(1-p)^{3 / 2}(1+p)^{1 / 2}(1+2 p)^{3 / 2} k^{2} \\
& +\frac{9}{8}(1-p)^{1 / 2}(1+p)^{3 / 2}(1+2 p)^{1 / 2} k^{2} .
\end{aligned}
$$

From (3.3), Theorem 1.1, part (a) and (2.3), we deduce

$$
\begin{aligned}
(1-p)^{1 / 2}(1+ & p)^{3 / 2}(1+2 p)^{1 / 2} k^{2} \\
= & \frac{8}{9} B\left(q^{8}\right)-\frac{10}{9} k^{2}-\frac{20}{9} p k^{2}+\frac{2}{3} p^{2} k^{2}+\frac{16}{9} p^{3} k^{2}-\frac{1}{9} p^{4} k^{2} \\
& +\frac{1}{3}(1-p)^{3 / 2}(1+p)^{1 / 2}(1+2 p)^{3 / 2} k^{2} \\
= & 1+\sum_{n=1}^{\infty}(-8 \sigma(n / 6)+48 \sigma(n / 12)-64 \sigma(n / 24)) q^{n},
\end{aligned}
$$

which is part (b).

Next, from (3.2), we obtain

$$
\begin{aligned}
&(1-p)^{3 / 4}(1+p)^{1 / 4}(1+2 p)^{9 / 4} k^{2}+(1-p)^{9 / 4}(1+p)^{3 / 4}(1+2 p)^{3 / 4} k^{2} \\
&= \frac{8}{3} A\left(q^{8}\right)-\frac{1}{6} k^{2}-\frac{1}{3} p k^{2}-p^{2} k^{2}-\frac{5}{6} p^{3} k^{2}+\frac{1}{12} p^{4} k^{2} \\
&-\frac{1}{2}(1-p)^{3 / 2}(1+p)^{1 / 2}(1+2 p)^{3 / 2} k^{2} .
\end{aligned}
$$

Then, appealing to (2.2), Theorem 1.1 and part (a), we deduce

$$
\begin{array}{r}
(1-p)^{3 / 4}(1+p)^{1 / 4}(1+2 p)^{9 / 4} k^{2}+(1-p)^{9 / 4}(1+p)^{3 / 4}(1+2 p)^{3 / 4} k^{2} \\
=2+\sum_{n=1}^{\infty}(-16 \sigma(n / 4)+96 \sigma(n / 8)-128 \sigma(n / 16)) q^{n} .
\end{array}
$$


Our next objective is to determine

$$
(1-p)^{3 / 4}(1+p)^{1 / 4}(1+2 p)^{9 / 4} k^{2}-(1-p)^{9 / 4}(1+p)^{3 / 4}(1+2 p)^{3 / 4} k^{2} .
$$

To do this we make use of the following two sums, which are closely related to $L(q)$ :

$$
L_{1,4}(q)=\sum_{\substack{n=1 \\ n \equiv 1(\bmod 4)}}^{\infty} \sigma(n) q^{n}, \quad L_{3,4}(q)=\sum_{\substack{n=1 \\ n \equiv 3(\bmod 4)}}^{\infty} \sigma(n) q^{n} .
$$

These sums have been evaluated by Cheng [9]. From [9, Theorem 3.5.1] we have

$$
\begin{aligned}
& L_{1,4}(q)=\frac{1}{32}(1-g)(1+g)^{3} w^{2}, \\
& L_{3,4}(q)=\frac{1}{32}(1-g)^{3}(1+g) w^{2},
\end{aligned}
$$

where

$$
g=(1-x)^{1 / 4}, \quad 1-x=\frac{(1-p)^{3}(1+p)}{(1+2 p)^{3}}, \quad w=(1+2 p)^{3 / 2} k
$$

(see [7]). From (3.6) and (3.7) we deduce

$$
L_{1,4}(q)-L_{3,4}(q)=\frac{1}{8} g w^{2}-\frac{1}{8} g^{3} w^{2} .
$$

By (3.8) we see that

$$
\begin{aligned}
& (1-p)^{3 / 4}(1+p)^{1 / 4}(1+2 p)^{9 / 4} k^{2}=g w^{2} \\
& (1-p)^{9 / 4}(1+p)^{3 / 4}(1+2 p)^{3 / 4} k^{2}=g^{3} w^{2} .
\end{aligned}
$$

From (3.5), (3.9), (3.10) and (3.11), we deduce

$$
\begin{gathered}
(1-p)^{3 / 4}(1+p)^{1 / 4}(1+2 p)^{9 / 4} k^{2}-(1-p)^{9 / 4}(1+p)^{3 / 4}(1+2 p)^{3 / 4} k^{2} \\
=g w^{2}-g^{3} w^{2}=8 L_{1,4}(q)-8 L_{3,4}(q) \\
=8 \sum_{\substack{n=1 \\
n \equiv 1(\bmod 4)}}^{\infty} \sigma(n) q^{n}-8 \sum_{\substack{n=1 \\
n \equiv 3(\bmod 4)}}^{\infty} \sigma(n) q^{n},
\end{gathered}
$$

that is,

$$
\begin{gathered}
(1-p)^{3 / 4}(1+p)^{1 / 4}(1+2 p)^{9 / 4} k^{2}-(1-p)^{9 / 4}(1+p)^{3 / 4}(1+2 p)^{3 / 4} k^{2} \\
=8 \sum_{\substack{n=1 \\
n \equiv 1(\bmod 2)}}^{\infty}(-1)^{(n-1) / 2} \sigma(n) q^{n}
\end{gathered}
$$


Adding and subtracting (3.4) and (3.12), we obtain

$$
\begin{aligned}
& (1-p)^{3 / 4}(1+p)^{1 / 4}(1+2 p)^{9 / 4} k^{2} \\
& \quad=1+\sum_{n=1}^{\infty}\left(4\left(\frac{-4}{n}\right) \sigma(n)-8 \sigma(n / 4)+48 \sigma(n / 8)-64 \sigma(n / 16)\right) q^{n}
\end{aligned}
$$

and

$$
\begin{aligned}
& (1-p)^{9 / 4}(1+p)^{3 / 4}(1+2 p)^{3 / 4} k^{2} \\
& =1+\sum_{n=1}^{\infty}\left(-4\left(\frac{-4}{n}\right) \sigma(n)-8 \sigma(n / 4)+48 \sigma(n / 8)-64 \sigma(n / 16)\right) q^{n},
\end{aligned}
$$

which are parts (c) and (d).

4. Proof of Theorem 1.3. Ramanujan's discriminant function $\Delta(q)$ is defined by

$$
\Delta(q)=q \prod_{n=1}^{\infty}\left(1-q^{n}\right)^{24} .
$$

Alaca, Alaca and Williams [5, eqns. (3.28)-(3.33)] have shown that

$$
\begin{aligned}
\Delta(q) & =2^{-4} p(1+p)^{4}(1-p)^{12}(1+2 p)^{3}(2+p)^{3} k^{12} \\
\Delta\left(q^{2}\right) & =2^{-8} p^{2}(1+p)^{2}(1-p)^{6}(1+2 p)^{6}(2+p)^{6} k^{12} \\
\Delta\left(q^{3}\right) & =2^{-4} p^{3}(1+p)^{12}(1-p)^{4}(1+2 p)(2+p) k^{12} \\
\Delta\left(q^{4}\right) & =2^{-16} p^{4}(1+p)(1-p)^{3}(1+2 p)^{3}(2+p)^{12} k^{12} \\
\Delta\left(q^{6}\right) & =2^{-8} p^{6}(1+p)^{6}(1-p)^{2}(1+2 p)^{2}(2+p)^{2} k^{12} \\
\Delta\left(q^{12}\right) & =2^{-16} p^{12}(1+p)^{3}(1-p)(1+2 p)(2+p)^{4} k^{12}
\end{aligned}
$$

Hence, by (1.5), (4.1), (4.3), (4.5), (4.6) and (4.7), we obtain

$$
\begin{aligned}
\sum_{n=1}^{\infty} a(n) q^{n} & =q \prod_{n=1}^{\infty}\left(1-q^{2 n}\right)\left(1-q^{4 n}\right)\left(1-q^{6 n}\right)\left(1-q^{12 n}\right) \\
& =\Delta\left(q^{2}\right)^{1 / 24} \Delta\left(q^{4}\right)^{1 / 24} \Delta\left(q^{6}\right)^{1 / 24} \Delta\left(q^{12}\right)^{1 / 24} \\
& =\frac{1}{4} p(2+p)(1-p)^{1 / 2}(1+p)^{1 / 2}(1+2 p)^{1 / 2} k^{2}
\end{aligned}
$$

which is part (a).

Also, by (1.6), (4.1), (4.2), (4.3), (4.5), (4.6) and (4.7), we have

$$
\begin{aligned}
\sum_{n=0}^{\infty} b(n) q^{n} & =\prod_{n=1}^{\infty}\left(1-q^{n}\right)^{-4}\left(1-q^{2 n}\right)^{10}\left(1-q^{4 n}\right)^{-4}\left(1-q^{6 n}\right)^{4}\left(1-q^{12 n}\right)^{-2} \\
& =\Delta(q)^{-1 / 6} \Delta\left(q^{2}\right)^{5 / 12} \Delta\left(q^{4}\right)^{-1 / 6} \Delta\left(q^{6}\right)^{1 / 6} \Delta\left(q^{12}\right)^{-1 / 12} \\
& =(1-p)^{1 / 4}(1+p)^{3 / 4}(1+2 p)^{7 / 4} k^{2}
\end{aligned}
$$


and, by (1.7), (4.1), (4.3), (4.4), (4.5), (4.6) and (4.7),

$$
\begin{aligned}
\sum_{n=0}^{\infty} c(n) q^{n} & =\prod_{n=1}^{\infty}\left(1-q^{2 n}\right)^{4}\left(1-q^{3 n}\right)^{-4}\left(1-q^{4 n}\right)^{-2}\left(1-q^{6 n}\right)^{10}\left(1-q^{12 n}\right)^{-4} \\
& =\Delta\left(q^{2}\right)^{1 / 6} \Delta\left(q^{3}\right)^{-1 / 6} \Delta\left(q^{4}\right)^{-1 / 12} \Delta\left(q^{6}\right)^{5 / 12} \Delta\left(q^{12}\right)^{-1 / 6} \\
& =(1-p)^{3 / 4}(1+p)^{1 / 4}(1+2 p)^{5 / 4} k^{2}
\end{aligned}
$$

which are parts (b) and (c).

Applying the change of sign principle (Theorem 2.3) to parts (b) and (c), we obtain

$$
\begin{aligned}
\sum_{n=0}^{\infty} b(n)(-q)^{n} & =(1-p(-q))^{1 / 4}(1+p(-q))^{3 / 4}(1+2 p(-q))^{7 / 4} k(-q)^{2} \\
& =\left(1+\frac{p}{1+p}\right)^{1 / 4}\left(1-\frac{p}{1+p}\right)^{3 / 4}\left(1-\frac{2 p}{1+p}\right)^{7 / 4}(1+p)^{4} k^{2} \\
& =(1-p)^{7 / 4}(1+p)^{5 / 4}(1+2 p)^{1 / 4} k^{2}
\end{aligned}
$$

and

$$
\begin{aligned}
\sum_{n=0}^{\infty} c(n)(-q)^{n} & =(1-p(-q))^{3 / 4}(1+p(-q))^{1 / 4}(1+2 p(-q))^{5 / 4} k(-q)^{2} \\
& =\left(1+\frac{p}{1+p}\right)^{3 / 4}\left(1-\frac{p}{1+p}\right)^{1 / 4}\left(1-\frac{2 p}{1+p}\right)^{5 / 4}(1+p)^{4} k^{2} \\
& =(1-p)^{5 / 4}(1+p)^{7 / 4}(1+2 p)^{3 / 4} k^{2}
\end{aligned}
$$

proving parts (d) and (e).

5. Proof of Theorem 1.4. By Theorem 1.3(a) and Table 1 we have

$$
\left(2 p+p^{2}\right)(1-p)^{1 / 2}(1+p)^{1 / 2}(1+2 p)^{1 / 2} k^{2}=\sum_{n=1}^{\infty} 4 a(n) q^{n} .
$$

By Theorem 1.2(a) we have

$$
\begin{aligned}
\left(1+p-2 p^{2}\right)(1-p)^{1 / 2}(1+p)^{1 / 2}(1+2 p)^{1 / 2} k^{2} & \\
= & 1+\sum_{n=1}^{\infty}(-8 \sigma(n / 2)+48 \sigma(n / 4)-64 \sigma(n / 8)) q^{n} .
\end{aligned}
$$

By Theorem 1.2(b) we have

$$
\begin{aligned}
(1+p)(1-p)^{1 / 2}(1+p)^{1 / 2}(1+2 p)^{1 / 2} k^{2} & \\
& =1+\sum_{n=1}^{\infty}(-8 \sigma(n / 6)+48 \sigma(n / 12)-64 \sigma(n / 24)) q^{n}
\end{aligned}
$$


The three assertions of Theorem 1.4 now follow using

$$
\begin{aligned}
1 & =-\frac{1}{2}\left(2 p+p^{2}\right)-\frac{1}{4}\left(1+p-2 p^{2}\right)+\frac{5}{4}(1+p), \\
p & =\frac{1}{2}\left(2 p+p^{2}\right)+\frac{1}{4}\left(1+p-2 p^{2}\right)-\frac{1}{4}(1+p), \\
p^{2} & =-\frac{1}{2}\left(1+p-2 p^{2}\right)+\frac{1}{2}(1+p) .
\end{aligned}
$$

6. Proofs of Theorems 1.6-1.25. Let $n \in \mathbb{N}$. We note that in the proofs of Theorems $1.6-1.8,1.11,1.14$ and 1.18 we set $n=2^{\alpha} N$, where $\alpha \in \mathbb{N}_{0}, N \in \mathbb{N}$ and $\operatorname{gcd}(N, 2)=1$, whereas in Theorems 1.9, 1.10, 1.12, $1.13,1.15-1.17$ and $1.19-1.24$ we set $n=2^{\alpha} 3^{\beta} N$, where $\alpha \in \mathbb{N}_{0}, \beta \in \mathbb{N}_{0}$, $N \in \mathbb{N}$ and $\operatorname{gcd}(N, 6)=1$. As $\sigma(n)$ is a multiplicative function of $n$, we have

$$
\begin{aligned}
\sigma\left(2^{\alpha} N\right) & =\sigma\left(2^{\alpha}\right) \sigma(N)=\left(2^{\alpha+1}-1\right) \sigma(N), \\
\sigma\left(2^{\alpha} 3^{\beta} N\right) & =\sigma\left(2^{\alpha}\right) \sigma\left(3^{\beta}\right) \sigma(N)=\frac{1}{2}\left(2^{\alpha+1}-1\right)\left(3^{\beta+1}-1\right) \sigma(N) .
\end{aligned}
$$

Proof of Theorem 1.6. We have

$$
\begin{aligned}
\sum_{n=0}^{\infty} N(1,1,1,1 ; n) q^{n} & =\varphi(q)^{4} & & (\text { by }(1.9)) \\
& =(1+2 p)^{3} k^{2} & & \text { (by Theorem 1.5(a)) } \\
& =1+\sum_{n=1}^{\infty}(8 \sigma(n)-32 \sigma(n / 4)) & & (\text { by Corollary 1.1(b)) }
\end{aligned}
$$

so for $n \in \mathbb{N}$,

$$
N(1,1,1,1 ; n)=8 \sigma(n)-32 \sigma(n / 4)= \begin{cases}8 \sigma(N) & \text { if } n \equiv 1(\bmod 2), \\ 24 \sigma(N) & \text { if } n \equiv 0(\bmod 2) .\end{cases}
$$

Proof of Theorem 1.7. We have

$$
\begin{aligned}
\sum_{n=0}^{\infty} N(1,1,1,4 ; n) q^{n} & =\varphi(q)^{3} \varphi\left(q^{4}\right) \quad(\text { by }(1.9)) \\
& =\frac{1}{2}(1+2 p)^{3} k^{2}+\frac{1}{2}(1-p)^{3 / 4}(1+p)^{1 / 4}(1+2 p)^{9 / 4} k^{2} \\
& \quad(\text { by Theorem } 1.5(\mathrm{a})(\mathrm{d})) \\
& 1+\sum_{n=1}^{\infty}\left(2\left(\frac{-4}{n}\right) \sigma(n)+4 \sigma(n)-20 \sigma(n / 4)\right. \\
& +24 \sigma(n / 8)-32 \sigma(n / 16)) q^{n}
\end{aligned}
$$

(by Corollary 1.1(b) and Theorem 1.2(c)), 
so for $n \in \mathbb{N}$,

$$
\begin{aligned}
N(1,1,1,4 ; n)= & \left(4+2\left(\frac{-4}{n}\right)\right) \sigma(n)-20 \sigma(n / 4)+24 \sigma(n / 8)-32 \sigma(n / 16) \\
= & \begin{cases}\left(4+2(-1)^{(N-1) / 2}\right) \sigma(N) & \text { if } n \equiv 1(\bmod 2), \\
12 \sigma(N) & \text { if } n \equiv 2(\bmod 4), \\
8 \sigma(N) & \text { if } n \equiv 4(\bmod 8), \\
24 \sigma(N) & \text { if } n \equiv 0(\bmod 8) .\end{cases}
\end{aligned}
$$

Proof of Theorem 1.8. We have

$$
\begin{aligned}
\sum_{n=0}^{\infty} N(1,1,2,2 ; n) q^{n} & =\varphi(q)^{2} \varphi\left(q^{2}\right)^{2} \quad(\text { by }(1.9)) \\
& =\frac{1}{2}(1+2 p)^{3} k^{2}+\frac{1}{2}(1-p)^{3 / 2}(1+p)^{1 / 2}(1+2 p)^{3 / 2} k^{2} \\
& =1+\sum_{n=1}^{\infty}(4 \sigma(n)-4 \sigma(n / 2)+8 \sigma(n / 4)-32 \sigma(n / 8)) q^{n}
\end{aligned}
$$

(by Corollary 1.1(b) and Theorem 1.2(a)),

so for $n \in \mathbb{N}$,

$$
\begin{aligned}
N(1,1,2,2 ; n) & =4 \sigma(n)-4 \sigma(n / 2)+8 \sigma(n / 4)-32 \sigma(n / 8) \\
& = \begin{cases}4 \sigma(N) & \text { if } n \equiv 1(\bmod 2), \\
8 \sigma(N) & \text { if } n \equiv 2(\bmod 4), \\
24 \sigma(N) & \text { if } n \equiv 0(\bmod 4) .\end{cases}
\end{aligned}
$$

Proof of Theorem 1.9. We have

$$
\begin{aligned}
\sum_{n=0}^{\infty} N(1,1,3,3 ; n) q^{n} & =\varphi(q)^{2} \varphi\left(q^{3}\right)^{2} \quad(\text { by }(1.9)) \\
& \left.=(1+2 p)^{2} k^{2} \quad \text { (by Theorem } 1.5(\mathrm{a})(\mathrm{c})\right) \\
& =1+\sum_{n=1}^{\infty}(4 \sigma(n)-8 \sigma(n / 2)-12 \sigma(n / 3)+16 \sigma(n / 4) \\
& +24 \sigma(n / 6)-48 \sigma(n / 12)) q^{n} \\
& \quad(\text { by Corollary } 1.1(\mathrm{a}))
\end{aligned}
$$

so for $n \in \mathbb{N}$,

$$
\begin{aligned}
N(1,1,3,3 ; n)= & 4 \sigma(n)-8 \sigma(n / 2)-12 \sigma(n / 3)+16 \sigma(n / 4) \\
& +24 \sigma(n / 6)-48 \sigma(n / 12) \\
= & \begin{cases}4 \sigma(N) & \text { if } n \equiv 1(\bmod 2), \\
4\left(2^{\alpha+1}-3\right) \sigma(N) & \text { if } n \equiv 0(\bmod 2) .\end{cases}
\end{aligned}
$$


Proof of Theorem 1.10. We have

$$
\begin{aligned}
\sum_{n=0}^{\infty} N(1,1,3,12 ; n) q^{n} & =\varphi(q)^{2} \varphi\left(q^{3}\right) \varphi\left(q^{12}\right) \quad(\text { by }(1.9)) \\
& =\frac{1}{2}(1+2 p)^{2} k^{2}+\frac{1}{2}(1-p)^{1 / 4}(1+p)^{3 / 4}(1+2 p)^{7 / 4} k^{2} \\
& \quad(\text { by Theorem } 1.5(\mathrm{a})(\mathrm{c})(\mathrm{f})) \\
& \left.+12 \sigma(n / 6)-24 \sigma(n / 12)+\frac{1}{2} b(n)\right) q^{n}
\end{aligned}
$$

(by Corollary 1.1(a) and Theorem 1.3(b)),

so for $n \in \mathbb{N}$,

$$
\begin{aligned}
N(1,1,3,12 ; n)= & 2 \sigma(n)-4 \sigma(n / 2)-6 \sigma(n / 3)+8 \sigma(n / 4) \\
& +12 \sigma(n / 6)-24 \sigma(n / 12)+\frac{1}{2} b(n) \\
= & \begin{cases}2 \sigma(N)+\frac{1}{2} b(n) & \text { if } n \equiv 1(\bmod 2), \\
2\left(2^{\alpha+1}-3\right) \sigma(N)+\frac{1}{2} b(n) & \text { if } n \equiv 0(\bmod 2) .\end{cases}
\end{aligned}
$$

Proof of Theorem 1.11. We have

$$
\begin{aligned}
& \sum_{n=0}^{\infty} N(1,1,4,4 ; n) q^{n}=\varphi(q)^{2} \varphi\left(q^{4}\right)^{2} \quad(\text { by }(1.9)) \\
&= \frac{1}{4}(1+2 p)^{3} k^{2}+\frac{1}{2}(1-p)^{3 / 4}(1+p)^{1 / 4}(1+2 p)^{9 / 4} k^{2} \\
&\left.+\frac{1}{4}(1-p)^{3 / 2}(1+p)^{1 / 2}(1+2 p)^{3 / 2} k^{2} \quad \text { (by Theorem } 1.5(\mathrm{a})(\mathrm{d})\right) \\
&= 1+\sum_{n=1}^{\infty}\left(\left(2+2\left(\frac{-4}{n}\right)\right) \sigma(n)-2 \sigma(n / 2)+8 \sigma(n / 8)-32 \sigma(n / 16)\right) q^{n}
\end{aligned}
$$

(by Corollary 1.1(b) and Theorem 1.2(a)(c)),

so for $n \in \mathbb{N}$,

$$
\begin{aligned}
N(1,1,4,4 ; n) & =\left(2+2\left(\frac{-4}{n}\right)\right) \sigma(n)-2 \sigma(n / 2)+8 \sigma(n / 8)-32 \sigma(n / 16) \\
& = \begin{cases}4 \sigma(N) & \text { if } n \equiv 1,2(\bmod 4), \\
0 & \text { if } n \equiv 3(\bmod 4), \\
8 \sigma(N) & \text { if } n \equiv 4(\bmod 8), \\
24 \sigma(N) & \text { if } n \equiv 0(\bmod 8) .\end{cases}
\end{aligned}
$$


Proof of Theorem 1.12. We have

$$
\begin{aligned}
\sum_{n=0}^{\infty} N & (1,1,6,6 ; n) q^{n}=\varphi(q)^{2} \varphi\left(q^{6}\right)^{2} \quad(\text { by }(1.9)) \\
= & \frac{1}{2}(1+2 p)^{2} k^{2}+\frac{1}{2}(1-p)^{1 / 2}(1+p)^{3 / 2}(1+2 p)^{3 / 2} k^{2} \\
= & \quad \text { by Theorem } 1.5(\mathrm{a})(\mathrm{e})) \\
& +\sum_{n=1}^{\infty}(2 \sigma(n)-2 \sigma(n / 2)-6 \sigma(n / 3)-4 \sigma(n / 4) \\
& +6 \sigma(n / 6)+16 \sigma(n / 8)+12 \sigma(n / 12)-48 \sigma(n / 24)+2 a(n)) q^{n}
\end{aligned}
$$

(by Corollary 1.1(a) and Corollary 1.2(a)),

so for $n \in \mathbb{N}$,

$$
\begin{aligned}
N(1,1,6,6 ; n)= & 2 \sigma(n)-2 \sigma(n / 2)-6 \sigma(n / 3)-4 \sigma(n / 4) \\
& +6 \sigma(n / 6)+16 \sigma(n / 8)+12 \sigma(n / 12)-48 \sigma(n / 24)+2 a(n) \\
= & \begin{cases}2 \sigma(N)+2 a(n) & \text { if } n \equiv 1(\bmod 2), \\
4 \sigma(N) & \text { if } n \equiv 2(\bmod 4), \\
4\left(2^{\alpha}-3\right) \sigma(N) & \text { if } n \equiv 0(\bmod 4),\end{cases}
\end{aligned}
$$

as $a(n)=0$ for $n \equiv 0(\bmod 2)$.

Proof of Theorem 1.13 and Theorem 1.25(b). We have

$$
\begin{aligned}
\sum_{n=0}^{\infty} N & (1,1,12,12 ; n) q^{n}=\varphi(q)^{2} \varphi\left(q^{12}\right)^{2} \quad(\text { by }(1.9)) \\
= & \frac{1}{4}(1+2 p)^{2} k^{2}+\frac{1}{2}(1-p)^{1 / 4}(1+p)^{3 / 4}(1+2 p)^{7 / 4} k^{2} \\
& \left.+\frac{1}{4}(1-p)^{1 / 2}(1+p)^{3 / 2}(1+2 p)^{3 / 2} k^{2} \quad \text { (by Theorem } 1.5(\mathrm{a})(\mathrm{f})\right) \\
= & +\sum_{n=1}^{\infty}(\sigma(n)-\sigma(n / 2)-3 \sigma(n / 3)-2 \sigma(n / 4)+3 \sigma(n / 6)+8 \sigma(n / 8) \\
& \left.+6 \sigma(n / 12)-24 \sigma(n / 24)+a(n)+\frac{1}{2} b(n)\right) q^{n}
\end{aligned}
$$

(by Corollary 1.1(a), Theorem 1.3(b) and Corollary 1.2(a)),

so for $n \in \mathbb{N}$,

$$
\begin{aligned}
N(1,1,12,12 ; n)= & \sigma(n)-\sigma(n / 2)-3 \sigma(n / 3)-2 \sigma(n / 4) \\
& +3 \sigma(n / 6)+8 \sigma(n / 8)+6 \sigma(n / 12) \\
& -24 \sigma(n / 24)+a(n)+\frac{1}{2} b(n)
\end{aligned}
$$




$$
= \begin{cases}\sigma(N)+a(n)+\frac{1}{2} b(n) & \text { if } n \equiv 1(\bmod 2), \\ 2 \sigma(N)+\frac{1}{2} b(n) & \text { if } n \equiv 2(\bmod 4), \\ 2\left(2^{\alpha}-3\right) \sigma(N)+\frac{1}{2} b(n) & \text { if } n \equiv 0(\bmod 4) .\end{cases}
$$

As $x^{2}+y^{2}+12 z^{2}+12 t^{2} \equiv x^{2}+y^{2} \equiv 0,1,2(\bmod 4)$, we have

$$
N(1,1,12,12 ; n)=0 \quad \text { if } n \equiv 3(\bmod 4) .
$$

Thus

$$
\sigma(n)+a(n)+\frac{1}{2} b(n)=0 \quad \text { if } n \equiv 3(\bmod 4),
$$

which is the assertion of Theorem 1.25(b).

Proof of Theorem 1.14. We have

$$
\begin{aligned}
& \sum_{n=0}^{\infty} N(1,2,2,4 ; n) q^{n}=\varphi(q) \varphi\left(q^{2}\right)^{2} \varphi\left(q^{4}\right) \quad(\text { by }(1.9)) \\
&= \frac{1}{4}(1+2 p)^{3} k^{2}+\frac{1}{4}(1-p)^{3 / 2}(1+p)^{1 / 2}(1+2 p)^{3 / 2} k^{2} \\
&+\frac{1}{4}(1-p)^{3 / 4}(1+p)^{1 / 4}(1+2 p)^{9 / 4} k^{2} \\
&+\frac{1}{4}(1-p)^{9 / 4}(1+p)^{3 / 4}(1+2 p)^{3 / 4} k^{2} \quad(\text { by Theorem } 1.5(\mathrm{a})(\mathrm{b})(\mathrm{d})) \\
&= 1+\sum_{n=1}^{\infty}(2 \sigma(n)-2 \sigma(n / 2)+8 \sigma(n / 8)-32 \sigma(n / 16)) q^{n}
\end{aligned}
$$

(by Corollary 1.1(b) and Theorem 1.2(a)(c)(d)),

so that for $n \in \mathbb{N}$,

$$
\begin{aligned}
N(1,2,2,4 ; n)= & 2 \sigma(n)-2 \sigma(n / 2)+8 \sigma(n / 8)-32 \sigma(n / 16) \\
& = \begin{cases}2 \sigma(N) & \text { if } n \equiv 1(\bmod 2), \\
4 \sigma(N) & \text { if } n \equiv 2(\bmod 4), \\
8 \sigma(N) & \text { if } n \equiv 4(\bmod 8), \\
24 \sigma(N) & \text { if } n \equiv 0(\bmod 8) .\end{cases}
\end{aligned}
$$

Proof of Theorem 1.15. We have

$$
\begin{aligned}
\sum_{n=0}^{\infty} N(1,2,3,6 ; n) q^{n}=\varphi(q) \varphi\left(q^{2}\right) \varphi\left(q^{3}\right) \varphi\left(q^{6}\right) \quad(\text { by }(1.9)) \\
=\frac{1}{2}(1+2 p)\left(1+p+p^{2}\right) k^{2}+\frac{1}{2}(1-p)^{1 / 2}(1+p)^{1 / 2}(1+2 p)^{3 / 2} k^{2}
\end{aligned}
$$

(by Theorem 1.5(a)(c) and (1.13)) 


$$
\begin{aligned}
= & +\sum_{n=1}^{\infty}(\sigma(n)-\sigma(n / 2)+3 \sigma(n / 3)+2 \sigma(n / 4)-3 \sigma(n / 6) \\
& -8 \sigma(n / 8)+6 \sigma(n / 12)-24 \sigma(n / 24)+a(n)) q^{n}
\end{aligned}
$$

(by Corollary 1.1(c) and Corollary 1.2(b)),

so for $n \in \mathbb{N}$,

$$
\begin{aligned}
N(1,2,3,6 ; n)= & \sigma(n)-\sigma(n / 2)+3 \sigma(n / 3)+2 \sigma(n / 4)-3 \sigma(n / 6) \\
& -8 \sigma(n / 8)+6 \sigma(n / 12)-24 \sigma(n / 24)+a(n) \\
= & \begin{cases}\left(3^{\beta+1}-2\right) \sigma(N)+a(n) & \text { if } n \equiv 1(\bmod 2), \\
2\left(3^{\beta+1}-2\right) \sigma(N) & \text { if } n \equiv 2(\bmod 4), \\
6\left(3^{\beta+1}-2\right) \sigma(N) & \text { if } n \equiv 0(\bmod 4),\end{cases}
\end{aligned}
$$

as $a(n)=0$ when $n$ is even.

Proof of Theorem 1.16. We have

$$
\begin{aligned}
& \sum_{n=0}^{\infty} N(1,3,3,4 ; n) q^{n}=\varphi(q) \varphi\left(q^{3}\right)^{2} \varphi\left(q^{4}\right) \quad(\text { by }(1.9)) \\
& \quad=\frac{1}{2}(1+2 p)^{2} k^{2}+\frac{1}{2}(1-p)^{3 / 4}(1+p)^{1 / 4}(1+2 p)^{5 / 4} k^{2}
\end{aligned}
$$

(by Theorem 1.5(a)(c)(d))

$$
=1+\sum_{n=1}^{\infty}(2 \sigma(n)-4 \sigma(n / 2)-6 \sigma(n / 3)+8 \sigma(n / 4)+12 \sigma(n / 6)
$$

$\left.-24 \sigma(n / 12)+\frac{1}{2} c(n)\right) q^{n} \quad($ by Corollary 1.1(a) and Theorem 1.3(c)), so for $n \in \mathbb{N}$,

$$
\begin{aligned}
N(1,3,3,4 ; n)= & 2 \sigma(n)-4 \sigma(n / 2)-6 \sigma(n / 3)+8 \sigma(n / 4)+12 \sigma(n / 6) \\
& -24 \sigma(n / 12)+\frac{1}{2} c(n) \\
= & \begin{cases}2 \sigma(N)+\frac{1}{2} c(n) & \text { if } n \equiv 1(\bmod 2), \\
2\left(2^{\alpha+1}-3\right) \sigma(N)+\frac{1}{2} c(n) & \text { if } n \equiv 0(\bmod 2) .\end{cases}
\end{aligned}
$$

Proof of Theorem 1.17 and Theorem 1.25(e). We have

$$
\begin{aligned}
& \sum_{n=0}^{\infty} N(1,3,4,12 ; n) q^{n}=\varphi(q) \varphi\left(q^{3}\right) \varphi\left(q^{4}\right) \varphi\left(q^{12}\right) \quad(\text { by }(1.9)) \\
&= \frac{1}{4}(1+2 p)^{2} k^{2}+\frac{1}{4}(1-p)^{1 / 4}(1+p)^{3 / 4}(1+2 p)^{7 / 4} k^{2} \\
&+\frac{1}{4}(1-p)^{3 / 4}(1+p)^{1 / 4}(1+2 p)^{5 / 4} k^{2}+\frac{1}{4}(1-p)(1+p)(1+2 p) k^{2}
\end{aligned}
$$

(by Theorem $1.5(\mathrm{a})(\mathrm{c})(\mathrm{d})(\mathrm{f})$ ) 


$$
\begin{aligned}
= & +\sum_{n=1}^{\infty}(\sigma(n)-3 \sigma(n / 2)-3 \sigma(n / 3)+8 \sigma(n / 4)+9 \sigma(n / 6) \\
& \left.-24 \sigma(n / 12)+\frac{1}{4} b(n)+\frac{1}{4} c(n)\right) q^{n}
\end{aligned}
$$

(by Corollary 1.1(a)(d) and Theorem 1.3(b)(c)),

so for $n \in \mathbb{N}$,

$$
\begin{aligned}
& N(1,3,4,12 ; n)=\sigma(n)-3 \sigma(n / 2)-3 \sigma(n / 3)+8 \sigma(n / 4)+9 \sigma(n / 6) \\
& -24 \sigma(n / 12)+\frac{1}{4} b(n)+\frac{1}{4} c(n) \\
& = \begin{cases}\sigma(N)+\frac{1}{4} b(n)+\frac{1}{4} c(n) & \text { if } n \equiv 1(\bmod 2), \\
\frac{1}{4} b(n)+\frac{1}{4} c(n) & \text { if } n \equiv 2(\bmod 4), \\
6\left(2^{\alpha-1}-1\right) \sigma(N)+\frac{1}{4} b(n)+\frac{1}{4} c(n) & \text { if } n \equiv 0(\bmod 4) .\end{cases}
\end{aligned}
$$

Now $x^{2}+3 y^{2}+4 z^{2}+12 t^{2} \equiv x^{2}+3 y^{2} \not \equiv 2(\bmod 4)$ so

$$
N(1,3,4,12 ; n)=0 \quad \text { if } n \equiv 2(\bmod 4) .
$$

Thus

$$
b(n)=-c(n) \quad \text { if } n \equiv 2(\bmod 4) .
$$

As

$$
\begin{aligned}
& x^{2}+y^{2}+12 z^{2}+12 t^{2} \equiv 2(\bmod 4) \Rightarrow x \equiv y(\bmod 2), \\
& x^{2}+y^{2}+12 z^{2}+12 t^{2}=2\left(\left(\frac{x+y}{2}\right)^{2}+\left(\frac{x-y}{2}\right)^{2}+6 z^{2}+6 t^{2}\right), \\
& 2\left(x^{2}+y^{2}+6 z^{2}+6 t^{2}\right)=(x+y)^{2}+(x-y)^{2}+12 z^{2}+12 t^{2}
\end{aligned}
$$

we have

$$
N(1,1,12,12 ; n)=N(1,1,6,6 ; n / 2) \quad \text { if } n \equiv 2(\bmod 4) .
$$

Appealing to Theorems 1.13 and 1.12, we obtain

$$
2 \sigma(N)+\frac{1}{2} b(n)=2 \sigma(N)+2 a(n / 2)
$$

so that

$$
b(n)=4 a(n / 2) \quad \text { if } n \equiv 2(\bmod 4) .
$$

This completes the proof of Theorem 1.25(e).

Proof of Theorem 1.18. We have

$$
\sum_{n=0}^{\infty} N(1,4,4,4 ; n) q^{n}=\varphi(q) \varphi\left(q^{4}\right)^{3}
$$




$$
\begin{aligned}
= & \frac{1}{8}(1+2 p)^{3} k^{2}+\frac{3}{8}(1-p)^{3 / 4}(1+p)^{1 / 4}(1+2 p)^{9 / 4} k^{2} \\
& +\frac{3}{8}(1-p)^{3 / 2}(1+p)^{1 / 2}(1+2 p)^{3 / 2} k^{2}+\frac{1}{8}(1-p)^{9 / 4}(1+p)^{3 / 4}(1+2 p)^{3 / 4} k^{2}
\end{aligned}
$$

(by Theorem $1.5(\mathrm{a})(\mathrm{d})$ )

$$
=1+\sum_{n=1}^{\infty}\left(\left(1+\left(\frac{-4}{n}\right)\right) \sigma(n)-3 \sigma(n / 2)+10 \sigma(n / 4)-32 \sigma(n / 16)\right) q^{n}
$$

(by Corollary 1.1(b) and Theorem 1.2(a)(c)(d)),

so for $n \in \mathbb{N}$,

$$
\begin{aligned}
N(1,4,4,4 ; n)= & \left(1+\left(\frac{-4}{n}\right)\right) \sigma(n)-3 \sigma(n / 2)+10 \sigma(n / 4)-32 \sigma(n / 16) \\
= & \begin{cases}2 \sigma(N) & \text { if } n \equiv 1(\bmod 4), \\
0 & \text { if } n \equiv 2,3(\bmod 4), \\
8 \sigma(N) & \text { if } n \equiv 4(\bmod 8), \\
24 \sigma(N) & \text { if } n \equiv 0(\bmod 8) .\end{cases}
\end{aligned}
$$

Proof of Theorem 1.19. We have

$$
\begin{array}{rl}
\sum_{n=0}^{\infty} N & N(1,4,6,6 ; n) q^{n}=\varphi(q) \varphi\left(q^{4}\right) \varphi\left(q^{6}\right)^{2} \quad(\text { by }(1.9)) \\
= & \frac{1}{4}(1+2 p)^{2} k^{2}+\frac{1}{4}(1-p)^{3 / 4}(1+p)^{1 / 4}(1+2 p)^{5 / 4} k^{2} \\
& +\frac{1}{4}(1-p)^{1 / 2}(1+p)^{3 / 2}(1+2 p)^{3 / 2} k^{2}+\frac{1}{4}(1-p)^{5 / 4}(1+p)^{7 / 4}(1+2 p)^{3 / 4} k^{2} \\
= & 1+\sum_{n=1}^{\infty}(\sigma(n)-\sigma(n / 2)-3 \sigma(n / 3)-2 \sigma(n / 4)+3 \sigma(n / 6)+8 \sigma(n / 8) \\
& \left.+6 \sigma(n / 12)-24 \sigma(n / 24)+a(n)+\frac{1}{4}\left(1+(-1)^{n}\right) c(n)\right) q^{n}
\end{array}
$$

(by Corollary 1.1(a), Theorem 1.3(c)(e) and Corollary 1.2(a)), so for $n \in \mathbb{N}$,

$$
\begin{aligned}
N(1,4,6,6 ; n)= & \sigma(n)-\sigma(n / 2)-3 \sigma(n / 3)-2 \sigma(n / 4)+3 \sigma(n / 6)+8 \sigma(n / 8) \\
& +6 \sigma(n / 12)-24 \sigma(n / 24)+a(n)+\frac{1}{4}\left(1+(-1)^{n}\right) c(n) \\
= & \begin{cases}\sigma(N)+a(n) & \text { if } n \equiv 1(\bmod 2), \\
2 \sigma(N)+\frac{1}{2} c(n) & \text { if } n \equiv 2(\bmod 4), \\
2\left(2^{\alpha}-3\right) \sigma(N)+\frac{1}{2} c(n) & \text { if } n \equiv 0(\bmod 4),\end{cases}
\end{aligned}
$$

as $a(n)=0$ for $n$ even. 
Proof of Theorem 1.20 and Theorem 1.25(a) $(f)(\mathrm{g})$. We have

$$
\begin{aligned}
\sum_{n=0}^{\infty} N(1, & , 12,12 ; n) q^{n}=\varphi(q) \varphi\left(q^{4}\right) \varphi\left(q^{12}\right)^{2} \quad(\text { by }(1.9)) \\
= & \frac{1}{8}(1+2 p)^{2} k^{2}+\frac{1}{8}(1-p)^{3 / 4}(1+p)^{1 / 4}(1+2 p)^{5 / 4} k^{2} \\
& +\frac{1}{8}(1-p)^{1 / 2}(1+p)^{3 / 2}(1+2 p)^{3 / 2} k^{2} \\
& +\frac{1}{8}(1-p)^{5 / 4}(1+p)^{7 / 4}(1+2 p)^{3 / 4} k^{2} \\
& +\frac{1}{4}(1-p)^{1 / 4}(1+p)^{3 / 4}(1+2 p)^{7 / 4} k^{2}+\frac{1}{4}(1-p)(1+p)(1+2 p) k^{2} \\
= & +\sum_{n=1}^{\infty}\left(\frac{1}{2} \sigma(n)-\frac{3}{2} \sigma(n / 2)-\frac{3}{2} \sigma(n / 3)+3 \sigma(n / 4)+\frac{9}{2} \sigma(n / 6)\right. \\
& +4 \sigma(n / 8)-9 \sigma(n / 12)-12 \sigma(n / 24) \\
& \left.+\frac{1}{2} a(n)+\frac{1}{4} b(n)+\frac{1}{8}\left(1+(-1)^{n}\right) c(n)\right) q^{n}
\end{aligned}
$$

(by Corollary 1.1(a)(d), Corollary 1.2(a) and Theorem 1.3(b)(c)(e)), so for $n \in \mathbb{N}$,

$$
\begin{aligned}
N(1,4,12,12 ; n)= & \frac{1}{2} \sigma(n)-\frac{3}{2} \sigma(n / 2)-\frac{3}{2} \sigma(n / 3)+3 \sigma(n / 4)+\frac{9}{2} \sigma(n / 6) \\
& +4 \sigma(n / 8)-9 \sigma(n / 12)-12 \sigma(n / 24) \\
& +\frac{1}{2} a(n)+\frac{1}{4} b(n)+\frac{1}{8}\left(1+(-1)^{n}\right) c(n) \\
= & \begin{cases}\frac{1}{2} \sigma(N)+\frac{1}{2} a(n)+\frac{1}{4} b(n) & \text { if } n \equiv 1(\bmod 2), \\
\frac{1}{4} b(n)+\frac{1}{4} c(n) & \text { if } n \equiv 2(\bmod 4), \\
2\left(2^{\alpha}-3\right) \sigma(N)+\frac{1}{4} b(n)+\frac{1}{4} c(n) & \text { if } n \equiv 0(\bmod 4),\end{cases}
\end{aligned}
$$

as $a(n)=0$ when $n$ is even.

As $x^{2}+4 y^{2}+6 z^{2}+6 t^{2} \equiv 1(\bmod 4)$ implies $z \equiv t(\bmod 2)$, and

$$
\begin{aligned}
x^{2}+4 y^{2}+6 z^{2}+6 t^{2} & =x^{2}+4 y^{2}+12\left(\frac{z+t}{2}\right)^{2}+12\left(\frac{z-t}{2}\right)^{2}, \\
x^{2}+4 y^{2}+12 z^{2}+12 t^{2} & =x^{2}+4 y^{2}+6(z+t)^{2}+6(z-t)^{2},
\end{aligned}
$$

we have

$$
N(1,4,6,6 ; n)=N(1,4,12,12 ; n) \quad \text { if } n \equiv 1(\bmod 4) .
$$

Appealing to Theorems 1.19 and 1.20 , we deduce

$$
\sigma(N)+a(n)=\frac{1}{2} \sigma(N)+\frac{1}{2} a(n)+\frac{1}{4} b(n) \quad \text { if } n \equiv 1(\bmod 4),
$$


so that

$$
b(n)=2 \sigma(N)+2 a(n) \quad \text { if } n \equiv 1(\bmod 4),
$$

which is Theorem 1.25(a).

As $x^{2}+4 y^{2}+12 z^{2}+12 t^{2} \equiv x^{2} \not \equiv 2,3(\bmod 4)$ we have

$$
N(1,4,12,12 ; n)=0 \quad \text { if } n \equiv 2,3(\bmod 4) \text {. }
$$

As $x^{2}+4 y^{2}+12 z^{2}+12 t^{2} \equiv 0(\bmod 4)$ implies $x \equiv 0(\bmod 2)$ we have

$$
N(1,4,12,12 ; n)=N(1,1,3,3 ; n / 4) \quad \text { if } n \equiv 0(\bmod 4) .
$$

Appealing to Theorems 1.20 and 1.9, we obtain

$2\left(2^{\alpha}-3\right) \sigma(N)+\frac{1}{4} b(n)+\frac{1}{4} c(n)= \begin{cases}4 \sigma(N) & \text { if } n \equiv 4(\bmod 8), \\ 4\left(2^{\alpha-1}-3\right) \sigma(N) & \text { if } n \equiv 0(\bmod 8),\end{cases}$

so that

$$
b(n)+c(n)= \begin{cases}8 \sigma(N) & \text { if } n \equiv 4(\bmod 8) \\ -24 \sigma(N) & \text { if } n \equiv 0(\bmod 8) .\end{cases}
$$

As $x^{2}+4 y^{2}+6 z^{2}+6 t^{2} \equiv 0(\bmod 4)$ implies $z \equiv t(\bmod 2)$, and

$$
\begin{aligned}
x^{2}+4 y^{2}+6 z^{2}+6 t^{2} & =x^{2}+4 y^{2}+12\left(\frac{z+t}{2}\right)^{2}+12\left(\frac{z-t}{2}\right)^{2}, \\
x^{2}+4 y^{2}+12 z^{2}+12 t^{2} & =x^{2}+4 y^{2}+6(z+t)^{2}+6(z-t)^{2},
\end{aligned}
$$

we have

$$
N(1,4,6,6 ; n)=N(1,4,12,12 ; n) \quad \text { if } n \equiv 0(\bmod 4) .
$$

Hence, by Theorems 1.19 and 1.20 , we have

$$
\begin{aligned}
2\left(2^{\alpha}-3\right) \sigma(N)+\frac{1}{2} c(n)=2\left(2^{\alpha}-3\right) \sigma(N)+\frac{1}{4} b(n)+\frac{1}{4} c(n) \\
\text { if } n \equiv 0(\bmod 4),
\end{aligned}
$$

so that

$$
b(n)=c(n) \quad \text { if } n \equiv 0(\bmod 4) .
$$

Thus

$$
\begin{array}{ll}
b(n)=c(n)=4 \sigma(N) & \text { if } n \equiv 4(\bmod 8), \\
b(n)=c(n)=-12 \sigma(N) & \text { if } n \equiv 0(\bmod 8) .
\end{array}
$$

This completes the proof of Theorem 1.25(f)(g). 
Proof of Theorem 1.21. We have

$$
\begin{aligned}
& \sum_{n=0}^{\infty} N(2,2,3,3 ; n) q^{n}=\varphi\left(q^{2}\right)^{2} \varphi\left(q^{3}\right)^{2} \quad(\text { by }(1.9)) \\
&= \frac{1}{2}(1+2 p)^{2} k^{2}+\frac{1}{2}(1-p)^{3 / 2}(1+p)^{1 / 2}(1+2 p)^{1 / 2} k^{2} \\
&= 1+\sum_{n=1}^{\infty}(2 \sigma(n)-2 \sigma(n / 2)-6 \sigma(n / 3) \\
&-4 \sigma(n / 4)+6 \sigma(n / 6)+16 \sigma(n / 8)+12 \sigma(n / 12) \\
&-48 \sigma(n / 24)-2 a(n)) q^{n}
\end{aligned}
$$

(by Corollary 1.1(a) and Corollary 1.2(c)),

so for $n \in \mathbb{N}$,

$$
\begin{aligned}
N(2,2,3,3 ; n)= & 2 \sigma(n)-2 \sigma(n / 2)-6 \sigma(n / 3)-4 \sigma(n / 4)+6 \sigma(n / 6) \\
& +16 \sigma(n / 8)+12 \sigma(n / 12)-48 \sigma(n / 24)-2 a(n) \\
= & \begin{cases}2 \sigma(N)-2 a(n) & \text { if } n \equiv 1(\bmod 2), \\
4 \sigma(N) & \text { if } n \equiv 2(\bmod 4), \\
4\left(2^{\alpha}-3\right) \sigma(N) & \text { if } n \equiv 0(\bmod 4) .\end{cases}
\end{aligned}
$$

Proof of Theorem 1.22. We have

$$
\begin{array}{rl}
\sum_{n=0}^{\infty} N & N(2,2,3,12 ; n) q^{n}=\varphi\left(q^{2}\right)^{2} \varphi\left(q^{3}\right) \varphi\left(q^{12}\right) \quad(\text { by }(1.9)) \\
= & \frac{1}{4}(1+2 p)^{2} k^{2}+\frac{1}{4}(1-p)^{3 / 2}(1+p)^{1 / 2}(1+2 p)^{1 / 2} k^{2} \\
& +\frac{1}{4}(1-p)^{1 / 4}(1+p)^{3 / 4}(1+2 p)^{7 / 4} k^{2}+\frac{1}{4}(1-p)^{7 / 4}(1+p)^{5 / 4}(1+2 p)^{1 / 4} k^{2} \\
= & +\sum_{n=1}^{\infty}(\sigma(n)-\sigma(n / 2)-3 \sigma(n / 3) \quad \text { by Theorem } 1.5(\mathrm{~b})(\mathrm{c})(\mathrm{f})) \\
& -2 \sigma(n / 4)+3 \sigma(n / 6)+8 \sigma(n / 8)+6 \sigma(n / 12) \\
& \left.-24 \sigma(n / 24)-a(n)+\frac{1}{4}\left(1+(-1)^{n}\right) b(n)\right) q^{n}
\end{array}
$$$$
\text { (by Theorem 1.5(b)(c)(f)) }
$$

(by Corollary 1.1(a), Corollary 1.2(c) and Theorem 1.3(b)(d)), so for $n \in \mathbb{N}$,

$$
\begin{aligned}
N(2,2,3,12 ; n)= & \sigma(n)-\sigma(n / 2)-3 \sigma(n / 3)-2 \sigma(n / 4)+3 \sigma(n / 6)+8 \sigma(n / 8) \\
& +6 \sigma(n / 12)-24 \sigma(n / 24)-a(n)+\frac{1}{4}\left(1+(-1)^{n}\right) b(n)
\end{aligned}
$$




$$
= \begin{cases}\sigma(N)-a(n) & \text { if } n \equiv 1(\bmod 2), \\ 2 \sigma(N)+\frac{1}{2} b(n) & \text { if } n \equiv 2(\bmod 4), \\ 2\left(2^{\alpha}-3\right) \sigma(N)+\frac{1}{2} b(n) & \text { if } n \equiv 0(\bmod 4),\end{cases}
$$

as $a(n)=0$ when $n$ is even.

Proof of Theorem 1.23 and Theorem 1.25(c). We have

$$
\begin{aligned}
& \sum_{n=0}^{\infty} N(3,3,4,4 ; n) q^{n}=\varphi\left(q^{3}\right)^{2} \varphi\left(q^{4}\right)^{2} \quad(\text { by }(1.9)) \\
&= \frac{1}{4}(1+2 p)^{2} k^{2}+\frac{1}{4}(1-p)^{3 / 2}(1+p)^{1 / 2}(1+2 p)^{1 / 2} k^{2} \\
&+\frac{1}{2}(1-p)^{3 / 4}(1+p)^{1 / 4}(1+2 p)^{5 / 4} k^{2} \quad(\text { by Theorem } 1.5(\mathrm{c})(\mathrm{d})) \\
&=+\sum_{n=1}^{\infty}(\sigma(n)-\sigma(n / 2)-3 \sigma(n / 3)-2 \sigma(n / 4)+3 \sigma(n / 6) \\
&\left.+8 \sigma(n / 8)+6 \sigma(n / 12)-24 \sigma(n / 24)-a(n)+\frac{1}{2} c(n)\right) q^{n}
\end{aligned}
$$

(by Corollary 1.1(a), Corollary 1.2(c) and Theorem 1.3(c)),

so for $n \in \mathbb{N}$,

$$
\begin{aligned}
N(3,3,4,4 ; n)= & \sigma(n)-\sigma(n / 2)-3 \sigma(n / 3)-2 \sigma(n / 4)+3 \sigma(n / 6) \\
& +8 \sigma(n / 8)+6 \sigma(n / 12)-24 \sigma(n / 24)-a(n)+\frac{1}{2} c(n) \\
= & \begin{cases}\sigma(N)-a(n)+\frac{1}{2} c(n) & \text { if } n \equiv 1(\bmod 2), \\
2 \sigma(N)+\frac{1}{2} c(n) & \text { if } n \equiv 2(\bmod 4), \\
2\left(2^{\alpha}-3\right) \sigma(N)+\frac{1}{2} c(n) & \text { if } n \equiv 0(\bmod 4) .\end{cases}
\end{aligned}
$$

As $3 x^{2}+3 y^{2}+4 z^{2}+4 t^{2} \not \equiv 1(\bmod 4)$ we have

$$
N(3,3,4,4 ; n)=0 \quad \text { if } n \equiv 1(\bmod 4) \text {. }
$$

Hence

$$
\sigma(N)-a(n)+\frac{1}{2} c(n)=0 \quad \text { if } n \equiv 1(\bmod 4)
$$

that is,

$$
c(n)=-2 \sigma(N)+2 a(n) \quad \text { if } n \equiv 1(\bmod 4)
$$

which is Theorem 1.25(c). 
Proof of Theorem 1.24 and Theorem 1.25(d). We have

$$
\begin{aligned}
\sum_{n=0}^{\infty} N & (3,4,4,12 ; n) q^{n}=\varphi\left(q^{3}\right) \varphi\left(q^{4}\right)^{2} \varphi\left(q^{12}\right) \quad(\text { by }(1.9)) \\
= & \frac{1}{8}(1+2 p)^{2} k^{2}+\frac{1}{4}(1-p)^{3 / 4}(1+p)^{1 / 4}(1+2 p)^{5 / 4} k^{2} \\
& +\frac{1}{8}(1-p)^{3 / 2}(1+p)^{1 / 2}(1+2 p)^{1 / 2} k^{2}+\frac{1}{8}(1-p)^{1 / 4}(1+p)^{3 / 4}(1+2 p)^{7 / 4} k^{2} \\
& +\frac{1}{4}(1-p)(1+p)(1+2 p) k^{2}+\frac{1}{8}(1-p)^{7 / 4}(1+p)^{5 / 4}(1+2 p)^{1 / 4} k^{2}
\end{aligned}
$$

(by Corollary 1.1(a)(d), Corollary 1.2(c) and Theorem 1.3(b)(c)(d))

$$
\begin{aligned}
= & 1+\sum_{n=1}^{\infty}\left(\frac{1}{2} \sigma(n)-\frac{3}{2} \sigma(n / 2)-\frac{3}{2} \sigma(n / 3)+3 \sigma(n / 4)+\frac{9}{2} \sigma(n / 6)+4 \sigma(n / 8)\right. \\
& \left.-9 \sigma(n / 12)-12 \sigma(n / 24)-\frac{1}{2} a(n)+\frac{1}{8}\left(1+(-1)^{n}\right) b(n)+\frac{1}{4} c(n)\right) q^{n},
\end{aligned}
$$

so for $n \in \mathbb{N}$,

$$
\begin{aligned}
N(3,4,4,12 ; n)= & \frac{1}{2} \sigma(n)-\frac{3}{2} \sigma(n / 2)-\frac{3}{2} \sigma(n / 3)+3 \sigma(n / 4)+\frac{9}{2} \sigma(n / 6) \\
& +4 \sigma(n / 8)-9 \sigma(n / 12)-12 \sigma(n / 24) \\
& -\frac{1}{2} a(n)+\frac{1}{8}\left(1+(-1)^{n}\right) b(n)+\frac{1}{4} c(n) \\
= & \begin{cases}\frac{1}{2} \sigma(N)-\frac{1}{2} a(n)+\frac{1}{4} c(n) & \text { if } n \equiv 1(\bmod 2), \\
\frac{1}{4} b(n)+\frac{1}{4} c(n) & \text { if } n \equiv 2(\bmod 4), \\
2\left(2^{\alpha}-3\right) \sigma(N)+\frac{1}{4} b(n)+\frac{1}{4} c(n) & \text { if } n \equiv 0(\bmod 4) .\end{cases}
\end{aligned}
$$

As $3 x^{2}+4 y^{2}+4 z^{2}+12 t^{2} \equiv 3 x^{2} \equiv 0,3(\bmod 4)$, we have

$$
N(3,4,4,12 ; n)=0 \quad \text { if } n \equiv 1,2(\bmod 4) .
$$
and

Finally, as $2 x^{2}+2 y^{2}+3 z^{2}+12 t^{2} \equiv 3(\bmod 4)$ implies $x \equiv y(\bmod 2)$,

$$
\begin{aligned}
& 2 x^{2}+2 y^{2}+3 z^{2}+12 t^{2}=3 z^{2}+4\left(\frac{x+y}{2}\right)^{2}+4\left(\frac{x-y}{2}\right)^{2}+12 t^{2}, \\
& 3 x^{2}+4 y^{2}+4 z^{2}+12 t^{2}=2(y+z)^{2}+2(y-z)^{2}+3 x^{2}+12 t^{2},
\end{aligned}
$$

we have

$$
N(2,2,3,12 ; n)=N(3,4,4,12 ; n) \quad \text { if } n \equiv 3(\bmod 4) .
$$

Appealing to Theorems 1.22 and 1.24, we obtain

$$
\sigma(N)-a(n)=\frac{1}{2} \sigma(N)-\frac{1}{2} a(n)+\frac{1}{4} c(n) \quad \text { if } n \equiv 3(\bmod 4),
$$


so that

$$
c(n)=2 \sigma(N)-2 a(n) \quad \text { if } n \equiv 3(\bmod 4),
$$

which is Theorem $1.25(\mathrm{~d})$.

The authors would like to thank an unknown referee for a number of valuable suggestions for improving the paper.

\section{References}

[1] A. Alaca, Ş. Alaca, M. F. Lemire and K. S. Williams, Jacobi's identity and representations of integers by certain quaternary forms, Int. J. Modern Math. 2 (2007), $143-176$.

[2] - - - - - - Theta function identities and representations by certain quaternary quadratic forms, Int. J. Number Theory, to appear.

[3] - , - - - - , The number of representations of a positive integer by certain quaternary quadratic forms, Int. J. Number Theory, to appear.

[4] A. Alaca, S.. Alaca and K. S. Williams, On the two-dimensional theta functions of the Borweins, Acta Arith. 124 (2006), 177-195.

[5] - - - - Evaluation of the convolution sums $\sum_{l+12 m=n} \sigma(l) \sigma(m)$ and $\sum_{3 l+4 m=n} \sigma(l) \sigma(m)$, Adv. Theoret. Appl. Math. $1(2006), 27-48$.

[6] - - - - - On the quaternary forms $x^{2}+y^{2}+z^{2}+5 t^{2}, x^{2}+y^{2}+5 z^{2}+5 t^{2}$ and $x^{2}+5 y^{2}+5 z^{2}+5 t^{2}$, JP J. Algebra Number Theory Appl., to appear.

[7] S. Alaca and K. S. Williams, Evaluation of the convolution sums $\sum_{l+6 m=n} \sigma(l) \sigma(m)$ and $\sum_{2 l+3 m=n} \sigma(l) \sigma(m)$, J. Number Theory 124 (2007), 491-510.

[8] P. Bachmann, Niedere Zahlentheorie, Chelsea, New York, 1968.

[9] N. Cheng, Convolution sums involving divisor functions, M.Sc. thesis, Carleton Univ., Ottawa, 2003.

[10] J. I. Deutsch, A quaternionic proof of the representation formula of a quaternary quadratic form, J. Number Theory 113 (2005), 149-179.

[11] R. Gongadze, On the representation of numbers by certain quadratic forms in four variables, Bull. Acad. Sci. Georgian SSR 28 (1962), 385-392 (in Russian).

[12] L. W. Griffiths, Generalized quaternion algebras and the theory of numbers, Amer. J. Math. 50 (1928), 303-314.

[13] - Representation of integers in the form $x^{2}+2 y^{2}+3 z^{2}+6 w^{2}$, ibid. 51 (1929), 61-66.

[14] H. D. Kloosterman, On the representation of numbers in the form $a x^{2}+b y^{2}+c z^{2}+$ $d t^{2}$, Proc. London Math. Soc. 25 (1926), 143-173.

[15] G. Köhler, On two of Liouville's quaternary forms, Arch. Math. (Basel) 54 (1990), 465-473.

[16] J. Liouville, Sur la forme $x^{2}+y^{2}+3\left(z^{2}+t^{2}\right)$, J. Math. Pures Appl. 5 (1860), 147-152.

[17] - Sur la forme $x^{2}+y^{2}+2\left(z^{2}+t^{2}\right)$, ibid. 5 (1860), 269-272.

[18] - Sur la forme $x^{2}+y^{2}+4\left(z^{2}+t^{2}\right)$, ibid. 5 (1860), 305-308.

[19] -, Sur la forme $x^{2}+3 y^{2}+4 z^{2}+12 t^{2}$, ibid. 6 (1861), 135-136.

[20] - Sur les deux formes $X^{2}+Y^{2}+Z^{2}+4 T^{2}, X^{2}+4 Y^{2}+4 Z^{2}+4 T^{2}$, ibid. 6 (1861), 440-448.

[21] - Sur la forme $x^{2}+2 y^{2}+2 z^{2}+4 t^{2}$, ibid. 7 (1862), 1-4.

[22] - Remarque nouvelle sur la forme $x^{2}+y^{2}+3\left(z^{2}+t^{2}\right)$, ibid. 8 (1863), 296 . 
[23] J. Liouville, Sur la forme $x^{2}+2 y^{2}+3 z^{2}+6 t^{2}$, ibid. 9 (1864), 299-312.

[24] - Sur les deux formes $x^{2}+y^{2}+6 z^{2}+6 t^{2}, 2 x^{2}+2 y^{2}+3 z^{2}+3 t^{2}$, ibid. 10 (1865), 359-360.

[25] Y. Martin, Multiplicative $\eta$-quotients, Trans. Amer. Math. Soc. 348 (1996), 48254856.

[26] T. Pepin, Étude sur quelques formules d'analyse utiles dans la théorie des nombres, Atti Accad. Pont. Nuovi Lincei 38 (1884-5), 139-196.

[27] - Sur quelques formes quadratiques quaternaires, J. Math. Pures Appl. 6 (1890), $5-67$.

[28] K. S. Williams, On the representations of a positive integer by the forms $x^{2}+y^{2}+$ $z^{2}+2 t^{2}$ and $x^{2}+2 y^{2}+2 z^{2}+2 t^{2}$, Int. J. Modern Math., to appear.

Centre for Research in Algebra and Number Theory

School of Mathematics and Statistics

Carleton University

Ottawa, Ontario, Canada K1S 5B6

E-mail: aalaca@connect.carleton.ca salaca@connect.carleton.ca mathieul@connect.carleton.ca

kwilliam@connect.carleton.ca

Received on 13.2.2007

and in revised form on 28.8.2007 